\title{
Hysteresis parameters and magnetic anisotropy of silicate-hosted magnetite exsolutions
}

Even S. Nikolaisen ${ }^{1}$, Richard Harrison ${ }^{1,2}$, Karl Fabian $^{1}$, Nathan Church $^{1}$, Suzanne A. McEnroe $^{1}$, Bjørn Eske Sørensen ${ }^{1}$, Christian Tegner ${ }^{3}$

${ }^{1}$ Norwegian University of Science and Technology (NTNU), S. P. Andersens veg 15a, 7031 Trondheim, Norway

${ }^{2}$ Department of Earth Sciences, University of Cambridge, Downing Street, Cambridge CB2 3EQ, UK

${ }^{3}$ Department of Geoscience, Aarhus University, Høegh-Guldbergs Gade 2, 8000 Aarhus, Denmark 


\section{Summary}

Anisotropy of remanent magnetization and magnetic susceptibility are highly sensitive and important indicators of geological processes which are largely controlled by mineralogical parameters of the ferrimagnetic fraction in rocks. To provide new physical insight into the complex interaction between magnetization structure, shape, and crystallographic relations, we here analyse "slice-and-view" focused-ion-beam (FIB) nano-tomography data with micromagnetic modelling and single crystal hysteresis measurements. The data sets consist of 68 magnetite inclusions in orthopyroxene $\left(M g_{60}\right)$ and 234 magnetite inclusions in plagioclase $\left(A n_{63}\right)$ were obtained on mineral separates from the Rustenburg Layered Suite of the Bushveld Intrusive Complex, South Africa. Electron backscatter diffraction was used to determine the orientation of the magnetite inclusions relative to the crystallographic directions of their silicate hosts. Hysteresis loops were calculated using the finite-element micromagnetics code MERRILL for each particle in 20 equidistributed field directions and compared with corresponding hysteresis loops measured using a vibrating sample magnetometer (VSM) on silicate mineral separates from the same samples. In plagioclase the ratio of remanent magnetization to saturation magnetization $\left(M_{r s} / M_{s}\right)$ for both model and measurement agree within $1.0 \%$, whereas the coercivity $\left(H_{c}\right)$ of the average modeled curve is $20 \mathrm{mT}$ lower than the measured value of $60 \mathrm{mT}$ indicating the presence of additional sources of high coercivity in the bulk sample. The VSM hysteresis measurements of the orthopyroxene were dominated by multi-domain (MD) magnetite, whereas the FIB location was chosen to avoid MD particles and thus contains only particles with diameters $<500 \mathrm{~nm}$ that are considered to be the most important carriers of paleomagnetic remanence. To correct for this sampling bias, measured MD hysteresis loops from synthetic and natural magnetites were combined with the average hysteresis loop from the MERRILL models of the FIB region. The result shows that while the modeled small-particle fraction only explains $6 \%$ of the best fit to the measured VSM hysteresis loop, it contributes $28 \%$ of the remanent magnetization. The modelled direction of maximal $M_{r s} / M_{s}$ in plagioclase is sub-parallel to [001] plag, whereas $H_{C}$ does not show a strong orientation dependence. The easy axis of magnetic remanence is in the direction of the magnetite population normal to (150) $)_{\text {plag }}$ and the maximum calculated susceptibility $\left(\chi^{*}\right)$ is parallel to $[010]_{\text {plag }}$. For orthopyroxene, the maximum $M_{r s} / M_{s}$, maximum $\chi^{*}$ and the easy axis of remanence is strongly correlated to the elongation axes of magnetite in the $[001]_{\mathrm{opx}}$ direction. The maximum $H_{c}$ is oriented along [100 $]_{\mathrm{opx}}$ and parallel to the minimum $\chi^{*}$, which reflects larger vortex nucleation fields when the applied field direction approaches 
the short axis. The maximum $H_{c}$ is therefore orthogonal to the maximum $M_{r s} / M_{s}$, controlled by axis-aligned metastable single-domain states at zero field. The results emphasize that the nature of anisotropy in natural magnetite does not just depend on the particle orientations, but on the presence of different stable and metastable domain states, and the mechanism of magnetic switching between them. Magnetic modelling of natural magnetic particles is therefore a vital method to extract and process anisotropic hysteresis parameters directly from the primary remanence carriers.

Keywords: Rock and mineral magnetism, Magnetic fabrics and anisotropy, Magnetic mineralogy and petrology, Numerical modelling, Microstructures.

\section{Introduction}

The Rustenburg Layered Suite of the Bushveld Intrusive Complex, South Africa is a 2055-2056 Ma [Scoates et al., 2021] layered intrusion covering an area of $65000 \mathrm{~km}^{2}$ and a maximum thickness of $7 \mathrm{~km}$ [Cawthorn et al., 2016], making it the largest and oldest known layered intrusion. Layered intrusions are distinguishable by sharp vertical differences between gravitationally segregated silicates, formed by crystal fractionation and settling of minerals in layers on the chamber floor. Geological maps [Wager and Brown, 1967] show the layered intrusive part of Bushveld Complex outcropping in four separate surface locations, the Northern, Western, South-Eastern and Eastern lobes [Letts et al., 2009]. These are internally connected and display distinct chemical similarities between their upper halves [Cawthorn et al., 1998; Webb et al., 2011]. The extensively studied Bushveld intrusion is considered a primary location for understanding the magmatic processes of deep seated magma chambers. Research interest is fueled by the economic enrichment of platinum group elements (PGE) that makes Bushveld the world's largest source of Pt and Pd mined every year [Crowson, 2001; Thormann et al., 2017].

Paleomagnetic studies [Feinberg et al., 2006; Hattingh, 1986] describe gabbronorites and anorthosites in the main zone of the Bushveld intrusion as displaying some of the most stable magnetic remanence properties encountered in ancient rocks [Feinberg et al., 2006]. This stable remanence is associated with magnetite that forms as exsolved or included particles in silicates like plagioclase [Davis, 1981; Feinberg et al., 2005; Usui et al., 2015; Sobolev, 1990] and pyroxene [Fleet et al., 1980; Frandsen et al., 2004; Renne et al., 2002; Feinberg 
et al., 2004] both within the Bushveld complex and in a wide range of other geologic settings.

These magnetite inclusions often occur as several micrometer long rods in multiple crystallographically defined directions. Given their common occurrence in nature, and their potential to carry reliable paleomagnetic information from ancient rocks [Bono et al., 2019], there is considerable interest in understanding the magnetic domain states of such inclusions [Nikolaisen et al., 2020] and the intrinsic magnetic anisotropy that arises from their non-random orientation distribution at the single-crystal scale. This anisotropy is of particular relevance when interpreting paleomagnetic measurements from single-crystal samples [Bono et al., 2019], as it has the potential to modify the relationship between the direction and intensity of paleomagnetic remanence and the ancient magnetizing field. More broadly, there is a fundamental need to understand the origin of anisotropy at the single-crystal scale, in order to relate bulk anisotropy measurements of igneous rocks to the mineralogical textures imparted by deformation and tectonic strain [Burton-Johnson et al., 2019]. Here we explore the physical origins of magnetic anisotropy from first principles using a combination of focused-ion-beam (FIB) "slice-andview" nanotomography and finite-element micromagnetic simulations. We show that the nature of the anisotropy depends not only on the orientation distribution of the magnetite rods, but also on the presence of different stable and metastable domain states and the mechanism of magnetic switching between them.

\section{Materials and Methods}

We here apply the general "slice-and-view" method for extracting data, processing the resulting image stack, and calculating micromagnetic models as it is described step-bystep in Nikolaisen et al. [2020]. For this study only few modifications to the method were introduced to increase the quality and accuracy of the results. Below only these changes are explained in sufficient detail to allow replication. All other details are described in Nikolaisen et al. [2020].

The most direct properties to compare modeled and measured data are the bulk average magnetic hysteresis parameters, $M_{s}$, saturation remanent magnetisation $M_{r s}$, coercivity $H_{c}$, and coercivity of remanence $H_{c r}$. In a bulk sample these properties are averaged over large particle ensembles that are usually randomly oriented in space, unless a lattice preferred orientation was generated during rock formation or later deformation. Each modeled curve represents a single field direction for a single particle, and the model results need to be averaged 
over both the particle ensemble and a sufficient number of field directions to compare them to the bulk measurements.

By this method it is possible to replicate equivalent hysteresis measurements based on FIB nanotomography and micromagnetic modeling [Nikolaisen et al., 2020]. Because the hysteresis curves for each particle is modelled for equidistributed external field directions, all magnetic hysteresis results can also be averaged for each individual direction over the whole particle ensemble, and then provide additional information about their magnetic anisotropy. The general method used to magnetically model hysteresis loops for a natural assemblage of magnetite from FIB nanotomography is documented in Nikolaisen et al. [2020]. Because these modelling results represent single silicate crystal hysteresis loops, they are here directly compared to VSM measurements of the corresponding silicate separates, even though these measurements represent much larger volumes. In addition, the modeled loops average only over smaller particle sizes which are within the FIB volume and can be modelled within a reasonable time. Therefore particles with an equivalent volume sphere diameter (EVSD) of $>500 \mu \mathrm{m}$ are excluded from the model.

\subsection{Sample}

The Rustenburg Layered Suite is subdivided into five zones, the Marginal (MZ), Lower (LZ), Critical (CZ), Main (MZ) and Upper (UZ). The B-16 specimen used in this study was collected on the Roossenekal Main Zone profile of the Bushveld eastern lobe $145 \mathrm{~m}$ below the pyroxenite marker [Cawthorn et al., 2016; Lundgaard et al., 2006]. Sample B-16 was chosen because magnetite is its dominant magnetic mineral composition and there are both plagioclase and pyroxene separates available allowing individual single crystal hysteresis loops to be measured. Bulk NRM and bulk magnetic susceptibility value are volume normalized and measured to $6.4 \mathrm{~A} / \mathrm{m}$ and 0.007 (SI) respectively for B-16 specifically. Petrographic analysis [Cawthorn et al., 2016] characterizes these samples as fine-grained gabbro norites with magnetite as the main opaque mineralization, appearing as an exsolved phase in both plagioclase and pyroxene. The incorporation of iron into the silicates was a primary liquidus process during the crystallization for plagioclase [Tegner and Cawthorn, 2010] and for pyroxene suggested to occur at primary subsolidus mineral formation, with magnetite exsolving at temperatures in the range of $600^{\circ} \mathrm{C}$ [Fleet et al., 1980] to $865^{\circ} \mathrm{C}$ [Feinberg et al., 2004]. 
Table 1. Plagioclase and orthopyxene compositions for sample B16 of the Bushveld Complex. Data compiled from Lundgaard et al. [2006] and Cawthorn et al. [2016]. Methods are described in Lundgaard [2003] and Lundgaard et al. [2006].

\begin{tabular}{c|ccc} 
Mineral & Plagioclase & Plagioclase & Orthopyroxene \\
\hline Method & XRF (bulk separate) & EMPA* & EMPA** \\
& & & \\
\hline
\end{tabular}

Major element compositions (wt\%)

\begin{tabular}{|c|c|c|c|}
\hline $\mathrm{SiO}_{2}$ & 52.86 & 52.21 & 52.25 \\
\hline $\mathrm{TiO}_{2}$ & 0.04 & & 0.15 \\
\hline $\mathrm{Al}_{2} \mathrm{O}_{3}$ & 29.14 & 28.79 & 0.76 \\
\hline $\mathrm{Cr}_{2} \mathrm{O}_{3}$ & & & 0.06 \\
\hline $\mathrm{Fe}_{2} \mathrm{O}_{3}$ & 0.24 & & 0.98 \\
\hline $\mathrm{FeO}$ & 0.50 & 0.52 & 23.63 \\
\hline $\mathrm{MnO}$ & 0.1 & & 0.52 \\
\hline$M g O$ & 0.27 & & 20.82 \\
\hline $\mathrm{CaO}$ & 12.26 & 12.38 & 1.01 \\
\hline $\mathrm{Na}_{2} \mathrm{O}$ & 4.40 & 4.09 & 0.015 \\
\hline $\mathrm{K}_{2} \mathrm{O}$ & 0.27 & 0.28 & \\
\hline $\mathrm{P}_{2} \mathrm{O}_{5}$ & 0.01 & & \\
\hline$L O I$ & 0.16 & & \\
\hline Sum & 100.16 & 98.28 & 100.19 \\
\hline$A n \%$ & 60.6 & $62.6 \pm 0.9$ & \\
\hline $\mathrm{Fe} \mathrm{O}_{\text {tot }}$ & 0.71 & & 24.51 \\
\hline$M g \#$ & & & $60.2 \pm 0.2$ \\
\hline
\end{tabular}

Trace element compositions by ICPMS (ppm)
$S r$
507.93
$B a$
100.21

\footnotetext{
$* 5 \mu \mathrm{m}$ beam $* * 2 \mu \mathrm{m}$ beam
} 
Plagioclase is labradorite $\left(A n_{62.6 \pm 0.9)}\right.$ [Lundgaard, 2003; Cawthorn et al., 2016], comprising elongated crystals up to to $1.5 \mathrm{~mm}$ in length with albite twins parallel to the direction of elongation. Some sections of the plagioclase show symplectite and inclusions of quartz. Fe-oxides are found as inclusions in multiple crystallographic orientations (Fig. A.5). The composition of plagioclase was determined both in situ (electron microprobe measurements with a 5 micron beam) and on the separated material (XRF measurements) at Aarhus University, Denmark. The two methods yield very similar results (Table 1), demonstrating that the plagioclase separate analysed in this study is very pure. The total iron content $(\mathrm{FeO}$ tot $)$ is $0.52 \mathrm{wt} \%$ for the in situ measurements and $0.71 \mathrm{wt} \%$ for the bulk separate. The higher $\mathrm{FeO}_{\mathrm{tot}}$ in the bulk separate may indicate that tiny amounts of oxide or pyroxene were included in this material. Alternatively, it indicates that the in situ measurements did not include exsolved magnetite inclusions. Pyroxene is mainly orthopyroxene $\left(M g_{60.2 \pm 0.2}\right)$ with clinopyroxenene occurring as larger discrete grains (labeled as Cpx in Fig. A.3). The orthopyroxene again show exsolution lamellae of inverted pigeonite (In-Pig in Fig. A.3). The composition of orthopyroxene was determined with a focused beam (ca. 2 micron) (Table 1). Clinopyroxene contains orthopyroxene exsolution lamellae with spinel. Fe-oxides hosted by the pyroxenes are observed in multiple relations. The largest occur as discrete grains of multi-domain magnetite $(300-500 \mu \mathrm{m})$ with oxy-exsolution of ilmenite lamellae and spinel (larger opaque grains in Fig. 1a). Within the orthopyroxene, there are multi-domain magnetite $(50-100 \mu \mathrm{m})$ inclusions with oxy-exsolved ilmenite lamellae (Fig. A.3 (Mag 1) and Fig. A.2c) oriented parallel to the inverted pigeonite lamellae. Occurring in local patches are magnetite exsolution lamellae (Fig. A.4 and A.3 (Mag 2)) in orthopyroxene that range between $0.2-20 \mu$ m wide and typically $<200 \mathrm{~nm}$ thick. Fig A.4 shows these magnetites to be in contact with thinner $(<200 \mathrm{~nm})$ inverted pigeonite lamellae. Whenever the exsolved inverted pigeonite lamellae are wider, the magnetites are also observed to be larger and oriented normal to the magnetite exsolved in orthopyroxene (Fig. A.4). Since we observe oxy-exsolved ilmenite in the larger magnetite inclusions, it is reasonable to assume that the magnetite lamellae in the pyroxene also contain ilmenite exsolution. While their small size makes it difficult to conclude, EDS does indicate trace values $<1 w t . \%$ Ti scattered in these magnetite lamellae. Unblocking temperatures measured by Letts et al. [2009] between $565-580{ }^{\circ} \mathrm{C}$ from similar samples in the eastern lobe Main Zone, suggest the main magnetic remanence carrier is low-Ti titanomagnetite or pure magnetite. Elsewhere in the pyroxene there are Fe-oxides that fill in cracks and at grain boundaries (Fig. A.2a and labeled Fe-oxide in Fig. A.3). 


\section{B-16}

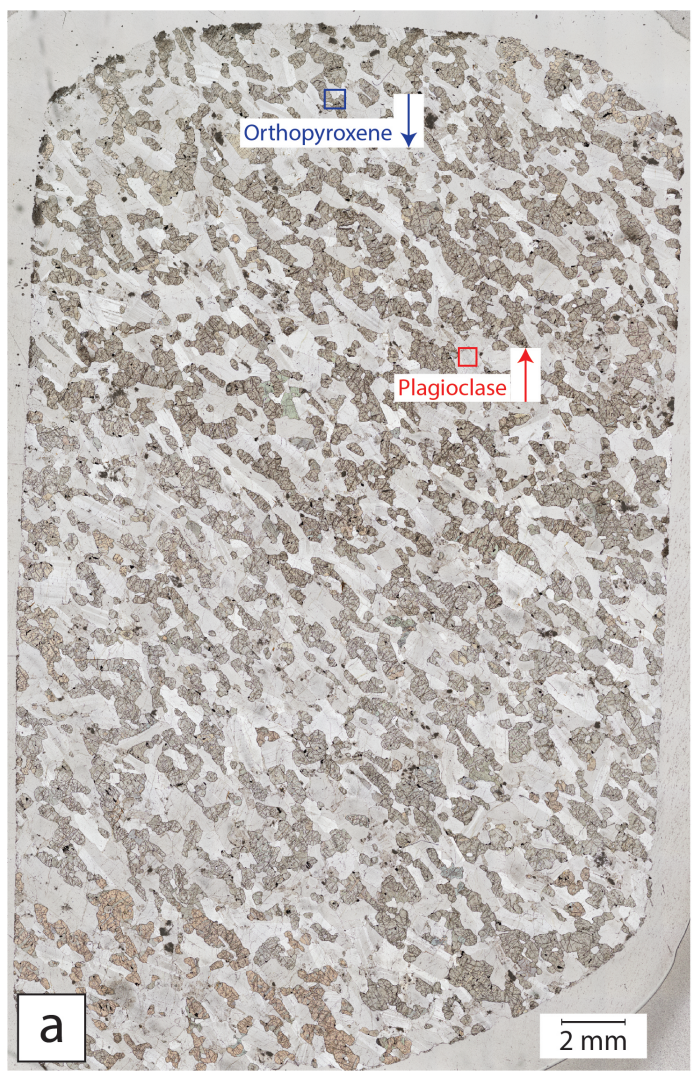

Plagioclase

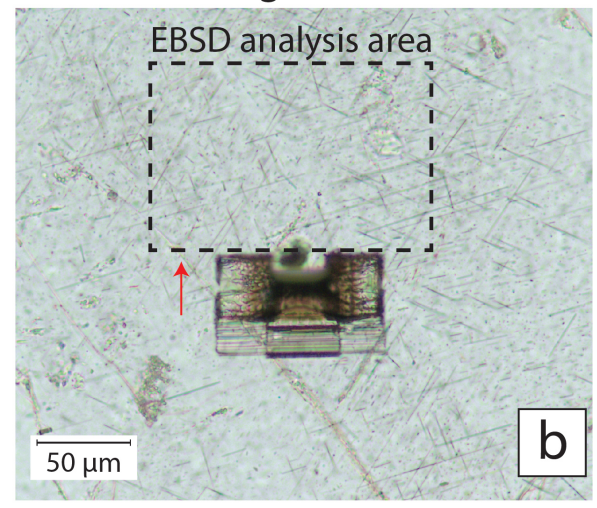

Orthopyroxene

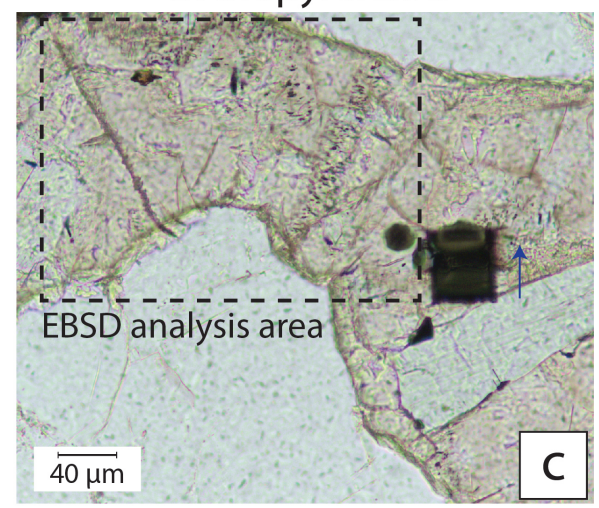

Figure 1. Optical plane polarized light scan of the B-16 thin section (a) showing the milling location in the plagioclase (b) and orthopyroxene (c). The red (plagioclase) and blue arrows (orthopyroxene) indicate the respective milling directions ( $F I B_{-Z}$-axis in Fig. 2 and 3). Note that the image in (c) is reversed with respect to (a). The black dashed boxes are the area analysed by EBSD. 


\subsection{Slice-and-View}

Raw data collection was performed using the Auto Slice \& View 4 software on an FEI Helios G4 UX Dual-Beam Focused-Ion-Beam (FIB) microscope at the NTNU NanoLab in Trondheim, Norway. The beam drift issues described by Nikolaisen et al. [2020] were reduced by avoiding magnifications higher than 2500x. Electron-beam imaging was performed using a through-lens detector (TLD) for the Backscattered-Electron (BSE) signal in immersion mode at $3 \mathrm{kV}$ and $0.4 \mathrm{nA}$. The milled volume from the plagioclase measures $27.93 \mu \mathrm{m}$ $\times 16.1 \mu \mathrm{m} \times 12.84 \mu \mathrm{m}\left(\mathrm{FIB}_{X}\right.$-axis $\times \mathrm{FIB}_{Y}$-axis $\times \mathrm{FIB}_{Z}$-axis) $($ Fig. 2$)$. This volume was reconstructed from 643 slices, with a slice thickness of $20 \mathrm{~nm}$. Each slice is a $1443 \times 894$ image with horizontal pixel size of $20.6 \mathrm{~nm}$. For the pyroxene the milled volume measures $25.71 \mu \mathrm{m} \times 14.31 \mu \mathrm{m} \times 6.32 \mu \mathrm{m}\left(\mathrm{FIB}_{X}\right.$-axis $\times \mathrm{FIB}_{Y}$-axis $\times \mathrm{FIB}_{Z}$-axis) (Fig. 3), reconstructed from 317 slices with a slice thickness of $20 \mathrm{~nm}$. Each slice is a 2113x1166 image with horizontal pixel size of $14.2 \mathrm{~nm}$.

\subsection{Image processing}

After stacking and aligning the raster images of the milled front faces, the individual slices were corrected to remove the shadow effect caused by the angle between the milled front face and e-beam. The darkening of the lower part of each slice due to this shadowing was removed by calculating the $\mathrm{Z}$ average of the entire stack. This $\mathrm{Z}$-average image predominantly reflects the voluminous host silicate and the instrumental shadowing, but also contains noise as well as brighter regions from areas where oxides persist over multiple slices. Therefore, a 2D polynomial surface was fitted to the $\mathrm{Z}$-average image and this surface is subtracted from each slice. Noise removal and smoothing was performed using a non-local means denoising Fiji plugin [Buades et al., 2011; Darbon et al., 2008]. Tilt correction for the $52^{\circ}$ angle between the ion- and electron-beam within the FEI software was unavailable for these samples and correction was done when recalculating pixel height $(\mathrm{H})$ in Paraview with $H_{\text {corrected }}=$ $H_{\text {Projected }} / \sin 52^{\circ}$. Further processing to generate each particle as a stereolithographic (STL) file an solidifying the meshes follows the exact process in Section 2.4 from Nikolaisen et al. [2020]. 


\subsection{Particle parameters}

Particle dimensions and orientations were calculated from the individual particle STL file through the two python packages numpy-stl (https://github.com/WoLpH/numpy-stl/) and trimesh [Dawson-Haggerty et al., 2019]. A particles direction is represented by the orientation of the elongation axis, calculated by the Eigen matrix of the inertia tensor. Dimensions were extracted by calculating the minimum volume bounding box.

\subsection{Micromagnetic modelling}

Micromagnetic modeling uses the finite element code MERRILL [Ó Conbhuí et al., 2018; Fabian and Shcherbakov, 2018]. Each particle's initial remanent domain state was determined by minimizing the total micromagnetic energy from a starting state of fully randomised spins. While Nikolaisen et al. [2020] did this once for each particle, here 20 minimizations were calculated and the lowest energy state was selected to indicate the initial domain state. Each particle's initial domain state of Single Domain (SD), Single Vortex (SV) and Multi Vortex (MV) was visualized using the open-source software ParaView (www.paraview.org) [Ayachit, 2015]. Simulating the 20 upper hysteresis-loop branches was replicated for each particle using the same method as Nikolaisen et al. [2020]. The applied field was swept from $350 \mathrm{mT}$ and $-350 \mathrm{mT}$ in $5 \mathrm{mT}$ steps. Hysteresis loops and backfield remanence curves were calculated for each 20 external field directions, chosen from a Fibonacci sphere (Mathematica code in the appendix) to achieve an approximately homogeneous distribution, presented in Fig. A.1 and "External field" column in Tab. A.1. The numerical average of the hysteresis loops and backfield curves over the 20 field directions for each mesh geometry approximately represents a random spherical ensemble of equal particles. Values of $M_{r s}, H_{c}$ and the calculated magnetic susceptibility at remanence $\left(\chi^{*}\right)$ were extracted from the average curves. $\left(\chi^{*}\right)$ is calculated by the first derivative of the hysteresis curve at $0 \mathrm{mT}$ and does not directly correspond to conventional measurements of the magnetic initial susceptibility $\chi_{0}$. The direction of magnetic remanence is a direct output from the micromagnetic modelling (in Cartesian coordinates) at each field increment and presented in this publication as an average for all particles at $0 \mathrm{mT}$. 


\subsection{Experimental magnetic measurements}

Plagioclase and orthopyroxene mineral separates were prepared by crushing bulk samples and isolating individual phases using a combination of magnetic separation and hand picking [Lundgaard et al., 2006], at the University of Aarhus. For measurement in the magnetometer, approximately $100 \mathrm{mg}$ of the separates were placed in gelatine capules, representing $10 \mathrm{~s}$ to $100 \mathrm{~s}$ of crystallites. Cyanoacrylate cement was used to immobilize the crystals of the plagioclase specimen and tightly pressed wadding for the orthopyroxene. Room-temperature hysteresis loops with maximum field $1 \mathrm{~T}$ were measured on a Princeton Measurement Corp. vibrating sample magnetometer at the Rock Magnetic Laboratory at NTNU and at the Institute for Rock Magnetism, University of Minnesota.

Hysteresis loops from a variety of coarse-grained magnetites were compiled to capture a range of multi-domain behaviors. A slice of natural, polycrystalline magnetite was measured in two orientations to observe the influence of sample shape anisotropy on the hysteresis loops, which are denominated MD2 nat1 and MD2 nat2 in this work. The specimen is dominantly magnetite in mm- to cm-scale grains with accessory serpentine and magnesite. Detailed chemical and magnetic characterization of this rock was reported by Pastore et al. [2019]. In addition, two synthetic samples produced using different methods were also included. Wright Industries W6 is a synthetic, hydrothermally precipitated magnetite powder with a mean particle size of $12.1 \mu \mathrm{m}$ [Krasa et al., 2003]. MD1 poly is a polycrystalline sample produced by sintering $\mathrm{Fe}_{2} \mathrm{O}_{3}$ at $1300{ }^{\circ} \mathrm{C}$ in the reducing conditions given by Dieckmann [1982]; full synthesis details in Church et al. [2011].

\subsection{EBSD}

The EBSD analysis was conducted on the Hitachi SU-6600 SEM at electron microscopy lab at IGP, NTNU, in low-vacuum mode using $20 \mathrm{kV}$ accelerating voltage, an extraction voltage of $1.70 \mathrm{kV}$. The samples were mounted on metal pedestals at standard eucentric height with a tilt angle of $70^{\circ}$ and working distance of $22.0 \mathrm{~mm}$. To avoid geometric trapezoidal scan distortions in the maps, a beam scan voltage correction was implemented using a EMTec LAMC-15 large area magnification calibration standard [S申rensen et al., 2020]. EBSD patterns were acquired using a NORDIF UF-1000 detector and the NORDIF 3.0 software. Indexing was done using the TSL-OIM 7.2 software at the Department of Material Science and Engineering, NTNU. EBSD data cleanup was done according to the procedures described 
in Sфrensen et al. [2020], checking the consistency of indexing and cleanup procedures. EBSD plotting was done using MTEX [Bachmann et al., 2010], version 5.7. The regions of the EBSD analysis are indicated by the black dotted boxes in Fig. 1b and 1c. The crystallographic directions of the silicate crystal are directly extrapolated to the milled volume by projecting the projecting all results parallel to $F I B-Y$ axis, then aligning $E B S D_{X}$ with $F I B_{X}$ and $E B S D_{Y}$ with $F I B_{Z}$.

The crystallographic relationship between magnetite needles and the plagioclase host are identified in $A n_{50}$ by Sobolev [1990] and $A n_{60-70}$ by Ageeva et al. [2020]. Exsolved magnetite in those studies was documented to crystallize in several orientations: one parallel to $[001]_{\text {plag }}$, two others in a plane perpendicular to $[001]_{\text {plag }}$ on the opposite sides of the $(010)_{\text {plag }}$ plane, almost coinciding with $(150)_{\text {plag }}$ and $(150)_{\text {plag }}$ planes [Sobolev, 1990], and two more orientations sub-parallel to $(112)_{\text {plag }}$ and $(\overline{3} 12)_{\text {plag }}$ planes [Ageeva et al., 2020]. For pyroxenes, ilmenite exsolution lamellae are present as coherent rods and blades where $(001)_{\mathrm{ilm}}$ is epitaxially intergrown on $(100)_{\mathrm{px}}$, and the $a$-axis of ilmenite is parallel to the $c$ axis of pyroxene [McEnroe et al., 2004]. Specifically magnetite is studied in detail by Fleet et al. $[1980]$ and reported to only exsolve as "Z" inclusions oriented parallel to the $[001]_{\mathrm{px}}$ axis, lying in the (100) opx plane [Kirby and Etheridge, 1981; Reiche and Bautsch, 1985; Champness and Lorimer, 1974].

It is important to emphasize that the dataset and sample were not primarily set up for these analyzes. The EBSD analysis is taken of the thin section surface within both the plagioclase and orthopyroxene crystal's in close vicinity, but not directly on the milled sections, because of the ion beam contamination (platinum and gallium) and destruction of the milling area. To achieve better alignment we recommend that the EBSD is measured before performing the FIB-nt. From the angled carbon rods added to the platinum cover, we can observe when stacking the slices that the amount of removed material during milling is not constant. By varying slice thickness we estimate a degree of misalignment at $\approx 5^{\circ}$ to a particle's phi and theta angle. Further development in interpolation techniques would improve the accuracy when integrating EBSD analysis. Because of the uncertainties explained above, we only used known magnetite orientations in silicates from highly detailed studies. Then, interpreting the population directions of magnetite in our FIB volumes, to the closest crystallographic direction know have exsolved iron oxides. 


\section{Results}

\subsection{Plagioclase}

This milled volume of plagioclase contain a total of 246 particles of magnetite and we were able to run hysteresis loops for 234 of these (red colored particles in Fig. 2). In this volume of plagioclase the magnetite forms three different patterns differentiated by shape and size. The crystallographic orientations are all interpreted from EBSD data and discussed below. The first and most noticeable are magnetite "plates" or "blades" that are a few to several micrometers long, $800 \mathrm{~nm}$ wide and $200 \mathrm{~nm}$ thick (grey in Fig. 2). The elongation axis for these magnetite blades are oriented normal to the $(150)_{\text {plag }}$ plane and sub-parallel to the $(\overline{3} 12)_{\text {plag }}$ plane. Because of their size these particles were not modelled in MERRILL. Furthermore, based on their morphology, we cannot definitely confirm that they are indeed magnetite. As they occur in the lower section of the milled volume, the BSE signal and the focus is weaker for the bottom third. The second set of particles are magnetite rods/needles with the major axis measuring up to several microns. These rods are very thin and appear sub-parallel to the $[001]_{\text {plag }}$ direction. The third set are smaller (sub $0.2 \mu \mathrm{m}$ ) prismatic magnetite particles with their elongation normal to the $(1 \overline{5} 0)_{\text {plag }}$ direction and sub-parallel to $(112)_{\text {plag }}$ plane. These inclusions are often associated with coexisting zircon and are in contact with an accessory mineral that has a lower BSE contrast than the plagioclase host (possibly quartz). While these three crystallographic orientations are the most populated there are 3-4 other orientations that can be observed by a few particles. Fig. A.5 provides a comparison between optically visible exsolutions and EBSD measurements of the area right above the milled site. Two of these additional directions are sub-parallel to the $(1 \overline{5} 0)_{\text {plag }}$ (red line in Fig. A.5) and $(150)_{\text {plag }}$ (orange line in Fig. A.5) planes. The particles in these other directions are modelled in MERRILL and added to the final results. However they will not be given any further consideration within this paper as their populations are too low to make a significant contribution to the total remanent magnetization.

\subsection{Orthopyroxene}

This milled volume of orthopyroxene contains 82 magnetite particles of which hysteresis loops were calculated for 68 . The majority of these particles are oblate $(a>b \gg c)$ This sample contains discrete grains of ortho- and clinopyroxene, with orthopyroxene as the most dominant pyroxene. inverted pigeonite (in-pig) exists as lamellae and smaller exsolved grains 


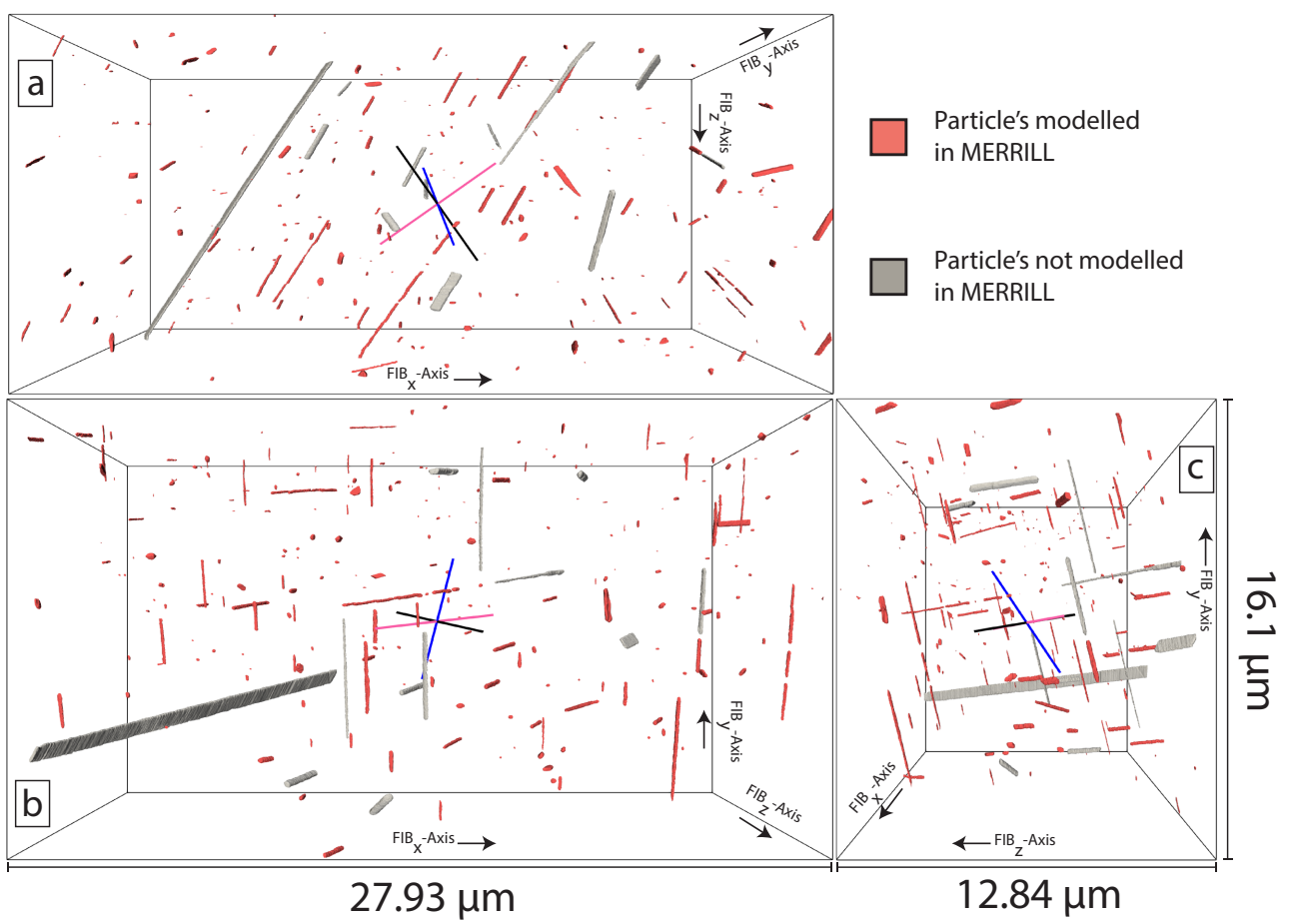

Figure 2. The three axis projections of the magnetite inclusions in the plagioclase with the dimensions $27.93 \mu \mathrm{m}$ (FIB $X_{X}$-axis), $16.1 \mu \mathrm{m}$ (FIB ${ }_{Y}$-axis) and $12.84 \mu \mathrm{m}$ (FIB $Z$-axis). a) Projection down the $\mathrm{FIB}_{Y}$-axis plane. b) Projection down the $\mathrm{FIB}_{Z}$-axis and c) projection down the $\mathrm{FIB}_{Y}$-axis. The black arrows illustrate the positive axis directions of the modelled volume. Projection a is oriented to view down at the surface of the milled volume, presenting the same viewing direction as when studying a thin section. The colored lines at the center of the volume are the crystallographic axis direction of the silicate host, derived from EBSD that is discussed below. Black is $[100]_{\text {plag }}$, magenta is $[010]_{\text {plag }}$ and blue $[001]_{\text {plag }}$. 


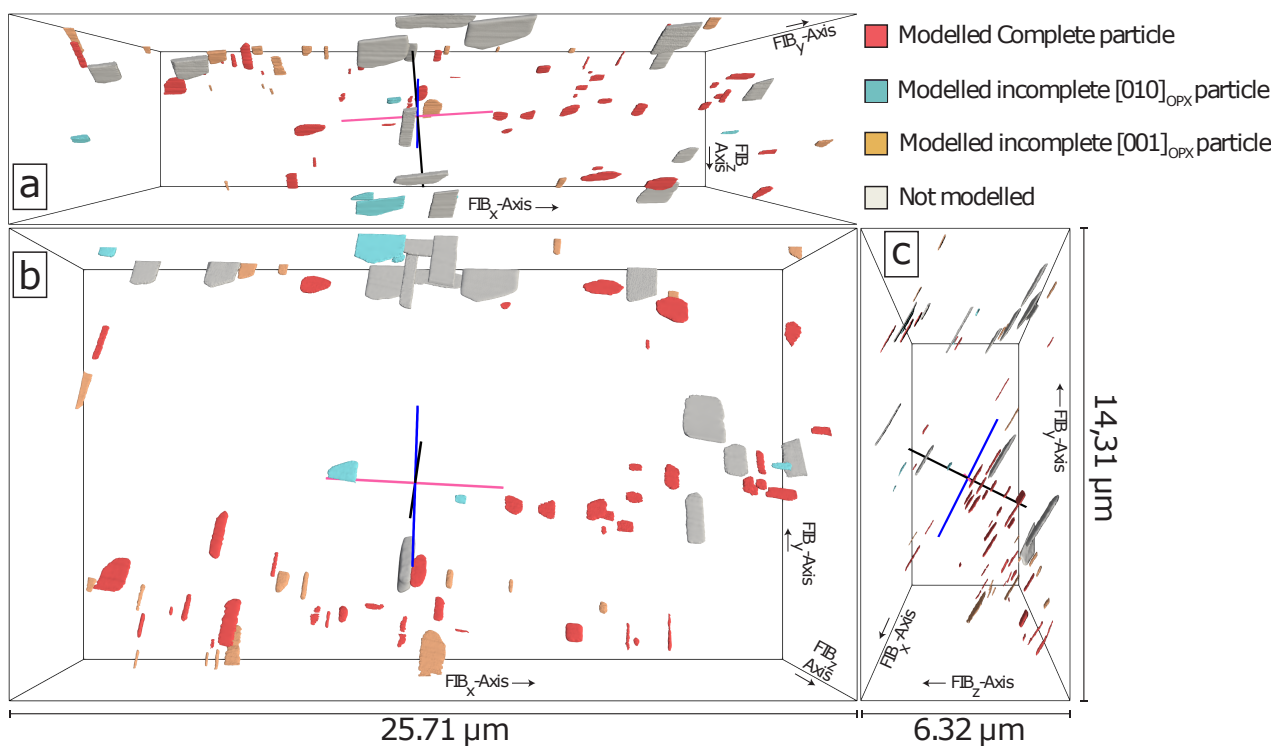

Figure 3. Three axis projection of the magnetite lamellea in orthopyroxene with the dimensions $25.71 \mu \mathrm{m}$ (FIB $X^{-}$-axis), $14.31 \mu \mathrm{m}$ (FIB ${ }_{Y}$-axis) and $6.32 \mu \mathrm{m}$ (FIB $Z_{Z}$-axis). a) Projection down the FIB $_{Y}$-axis, b) projection down the $\mathrm{FIB}_{Z}$-axis and c) projection down the $\mathrm{FIB}_{X}$-axis. The black arrows illustrate the positive axes directions of the modelled volume. Projection a is oriented to view down at the surface of the milled volume, presenting the same viewing direction as when studying a thin section. The colored lines at the center of the volume are the crystallographic axis direction of the silicate host, derived from EBSD that is discussed below. Black shows the $[100]_{\mathrm{opx}}$, magenta the $[010]_{\mathrm{opx}}$ and blue the $[001]_{\mathrm{opx}}$ axis direction.

within orthopyroxene. Magnetite is commonly observed as larger oxy-exolution lamellae, but also irregularly as exsolution lamellae in orthopyroxene. They are always in contact with thin $(<200 \mathrm{~nm})$ in-pig lamellae and elongated in the $[001]_{\mathrm{opx}}$ direction and flattened parallel to the $(100)_{\text {opx }}$ plane. The determined crystallographic orientations from our EBSD analysis, identified exsolved oxide and inverted pigeonite lamellae in the (100) opx plane . From Fig. 3 it can be seen that 24 particles (colored teal and yellow) are truncated by the milling volume, and that these truncated particles contribute to the population of $[010]_{\mathrm{opx}}$ oriented particles. Although these truncated particles were simulated, their contribution to the total moment are less compared to the 44 complete particles (red in Fig. 3).

\subsection{Micromagnetic properties}

For the plagioclase volume, the 234 modelled particles can be divided into 168 singledomain (SD), 60 single vortex (SV) and 6 multi-vortex (MV). Within the orthopyroxene 
Table 2. Hysteresis parameters for the magnetic models in Fig. 4. The average $H_{c}(\mathrm{mT})$ and $M_{r s} / M_{s}$ is representative for the specific particle over all 20 external fields. The two last columns represent the direction of the field that resulted in the maximum $H_{c}(\mathrm{mT})$ and $M_{r s} / M_{s}$. Field direction numbers refer to Tab. A.1 where the corresponding coordinates are listed in columns "External field".

\begin{tabular}{l|c|cc|cc|cc} 
Particle & $\begin{array}{c}\text { Domain } \\
\text { state }\end{array}$ & $\begin{array}{c}\text { Average } \\
H_{c}(\mathrm{mT})\end{array}$ & $M_{r s} / M_{s}$ & direction (Nr) & $H_{c}(\mathrm{mT})$ & $\begin{array}{c}\text { Field } \\
\text { direction (Nr) }\end{array}$ & $M_{r s} / M_{s}$ \\
\hline OPX026 & $\mathrm{SD}$ & 31.7 & 0.46 & 17 & 57 & 6 & 0.89 \\
OPX058 & $\mathrm{SV}$ & 13.5 & 0.52 & 15 & 39 & 14 & 0.94 \\
OPX036 & $\mathrm{MV}$ & 14.2 & 0.58 & 20 & 52 & 14 & 0.945 \\
OPX071 & $\mathrm{MV}$ & 15.4 & 0.50 & 15 & 35 & 9 & 0.94
\end{tabular}

volume there are $24 \mathrm{SD}, 27 \mathrm{SV}$ and $17 \mathrm{MV}$, based on the lowest of 20 energy states minimized from a random seed. A selection of modelled particles from the orthopyroxene is visualized in Fig. 4 with magnetic parameters in Table 2 for three different remanent states: column 1) the domain state representing the lowest of 20 energy states minimized from a random seed, column 2) the saturation remanent state obtained after applying a saturating field in the direction (Fig. A.1) that yielded the max $H_{c}$ and column 3) the saturation remanent state obtained after applying a saturating field in the direction that yielded the $\max M_{r s} / M_{s}$. OPX026 is an SD particle where the maximum $H_{c}$ is when fields are at a $40^{\circ}$ angle to the particle elongation axis. The maximum $M_{r s} / M_{s}$ is found for external fields applied at a low angle $\left(\leq 20^{\circ}\right)$ to the elongation axis. For SV and MV particles OPX058, 036 and 071, the field direction yielding $\max H_{c}$ is aligned with the shortest axis and the field direction yielding $\max M_{r s} / M_{S}$ is in the direction of the elongation axis.

Fig. 5 and Tab. A.1 compare the hysteresis curves and micromagnetic properties for both orthopyroxene (a) and plagioclase (b), where each hysteresis curve represents the sum of loops for all particles for a given field direction. Two hysteresis curves representing extracted single silicate crystals of orthopyroxene and plagioclase, measured on a vibrating sample magnetometer (VSM) are shown in blue for orthopyroxene (Fig. 6a) and plagioclase (Fig. 6b). The red curves in Fig. 6 are the result of summing all calculated hysteresis loops over all the external field directions, weighted by $M_{S}$, for orthopyroxene (a) and plagioclase (b). Using the hysteresis loops of pure MD magnetite in Fig. 7 as a reference, an estimate of the proportions of MD vs SD-MV particles in the orthopyroxene separates can be made using a 


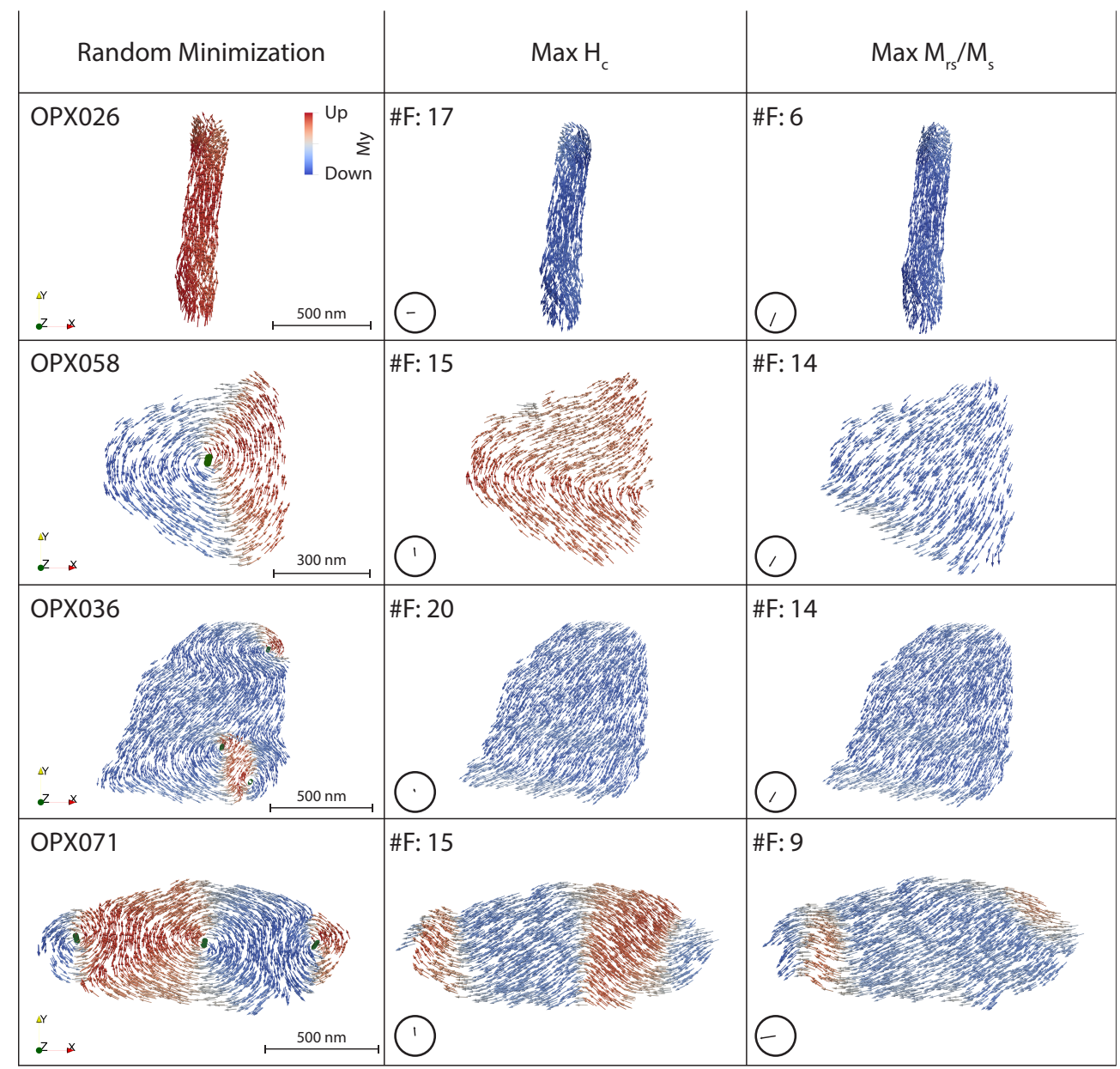

Figure 4. Micromagnetic models of selected particles from the orthopyroxene. The random minimization states (left column) are the remanent domain states having the lowest of 20 energy minimizations from a random seed. The maximum $H_{c}$ (central column) and maximum $M_{r s} / M_{s}$ (right column) states are saturation remanent states calculated from the hysteresis loops for individual external field directions (\#F) that yielded the maximum coercivity and saturation remanence, respectively (Fig. A.1). The $\mathrm{FIB}_{X}$ and $\mathrm{FIB}_{Y}$ direction of \#F is visually represented as a stereographic projection of the upper hemisphere, for each particle by the arrow at the bottom left corner. All external fields have a positive $\mathrm{FIB}_{Z}$ direction oriented out of the page. An accompanied figure for particles in the plagioclase can be found in Fig. A.7 

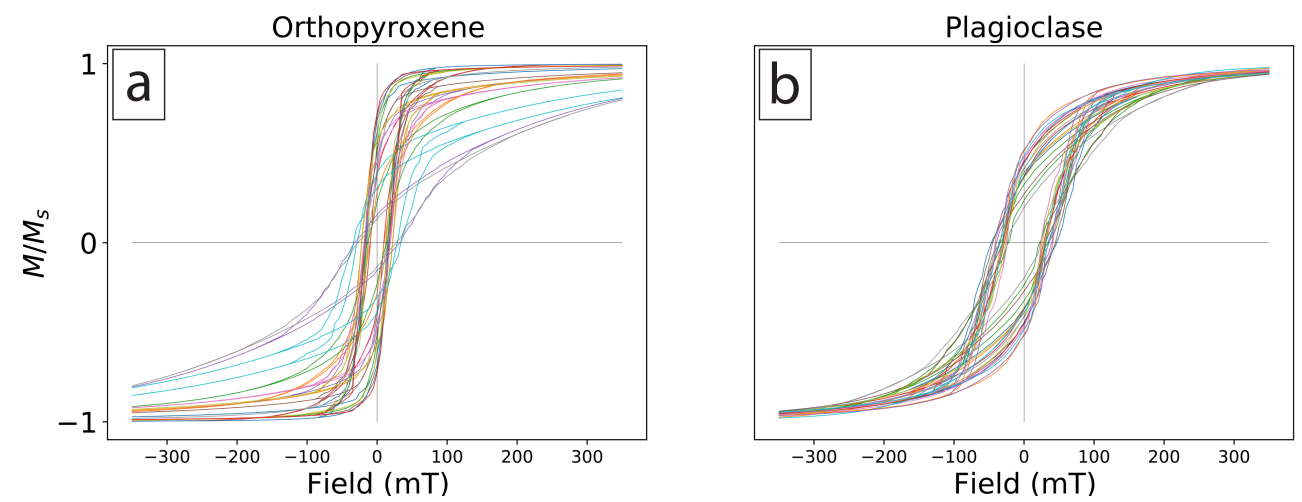

Figure 5. Magnetic hysteresis curves for all particles orthopyroxene (a) and in plagioclase (b) for the individual fields direction from -350 to $350 \mathrm{mT}$. Each loop is generated by summarizing all particle's hysteresis for a different applied field. The resulting $H_{c}$ and $M_{r s} / M_{S}$ for each loop is presented in Tab. A.1
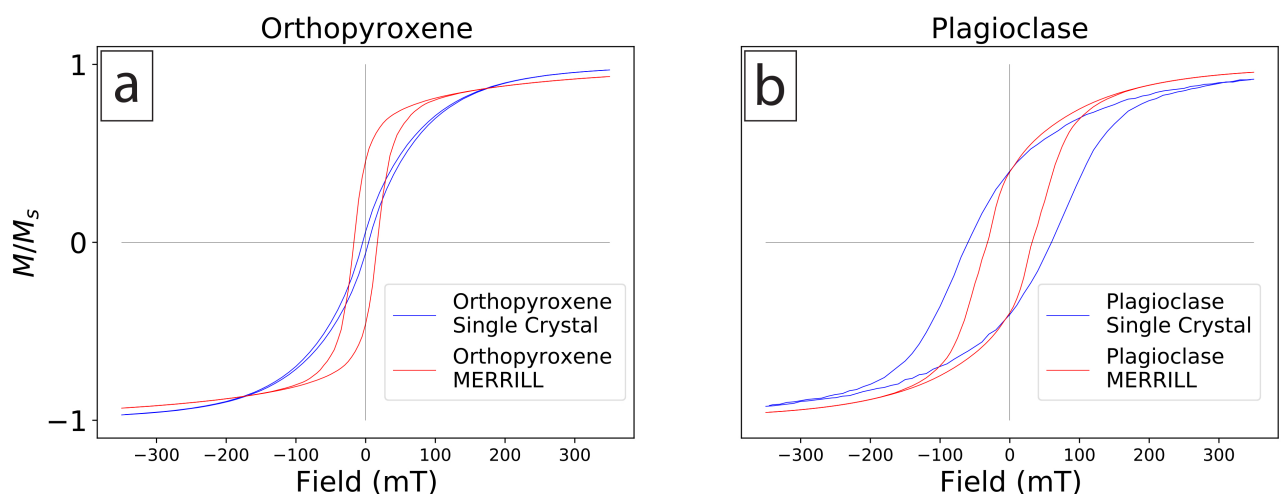

Figure 6. Magnetic hysteresis curves for fields from -350 to $350 \mathrm{mT}$ of a single silicate crystal (blue) and MERRILL calculations (red) of (a) orthopyroxene and (b) plagioclase from B16 sample. $H_{c}$ and $M_{r s} / M_{s}$ is presented in Tab. 3 
Table 3. Magnetic properties for the plagioclase and orthopyroxene single crystal measurements and the milled volumes calculated with MERRILL. For the crystals, the saturation magnetization $M_{S}$ is calculated by dividing the crystal saturation moment $\left(\mu_{s}\right)$ by the crystal volume $V$. The susceptibility $\chi^{*}$ is the slope of the upper hysteresis branch at zero field.

\begin{tabular}{lcccccc} 
Sample & $V\left(\mu \mathrm{m}^{3}\right)$ & $M_{r s} / M_{s}$ & $\mu_{s}\left(\mu \mathrm{Am}^{2}\right)$ & $H_{c}(\mathrm{mT})$ & $M_{s}(\mathrm{~A} / \mathrm{m})$ & $\chi^{*}(\mathrm{SI})$ \\
\hline PLAG single crystal & $4.26 \times 10^{-2}$ & 0.40 & 2.2 & 65.04 & 51.6 & 2.95 \\
PLAG MERRILL & $5.8 \times 10^{-9}$ & 0.39 & $5.44 \times 10^{-7}$ & 36.97 & 93.8 & 4.3 \\
OPX single crystal & $2.45 \times 10^{-2}$ & 0.06 & 54.7 & 9.26 & 2234 & 7.6 \\
OPX MERRILL & $2.33 \times 10^{-9}$ & 0.46 & $4.1 \times 10^{-7}$ & 21.9 & 177 & 10.9
\end{tabular}

non-negative least-squares approach [Lawson and Hanson, 1995] which combines different weight contributions of the MERRILL and MD curves to obtain a best fit calculation that resulted in a hysteresis curve (Fig. 7b) composed of 48\% W6 spec 7, 29.4\% MD2 nat1, 16.7\% MD2 nat2 and 5.6\% MERRILL (Tab. 4). The total error between the optimized and measured single crystal hysteresis loops shows an average deviation between the two curves of $1.64 \%$ (red curve in Fig. 7b). The MD curves in Fig. 7a have a variable rate in approach to saturation. As the gradient (1/N) [Stacey and Banerjee, 1974] of the hysteresis slope is dependent on the internal demagnetization factor $(\mathrm{N})$, the difference in approach to saturation between the MERRILL and single crystal curve is the higher demagnetization factor of the larger MD magnetite. Calculating the fraction of remanence contributed by each of the MD and SD-MV fractions yields 41.7\% W6 spec 7, 17.2\% MD2 nat1, 12.6\% MD2 nat2 and $28.4 \%$ MERRILL (Tab. 4).

The highest calculated MERRILL hysteresis parameters in the orthopyroxene, $M_{r s} / M_{S}$ values of $0.68-0.72$ in Tab. A.1 agree well with the magnetite elongation directions, which are sub-parallel to [001 $]_{\text {opx }}$ (Fig. 3c). They correspond to field directions 6, 11 and 14 (Fig. A.1).The lowest $M_{r s} / M_{s}$ values of $0.132-0.153$ are observed for field directions normal to the flattened blade surfaces of the magnetite lamellae and sub-parallel to [100] $]_{\mathrm{opx}}$ at the field directions 13,15 , and 18. $H_{c}$ values show a different trend: intermediate values of $\approx 17 \mathrm{mT}$ are observed parallel to the elongation direction of magnetite, maximum values of 27-32 mT are observed normal to the flattened blade surfaces, and minimum values of $9-10 \mathrm{mT}$ are observed along the intermediate length axis. For $\chi^{*}$ the maximum of 17 (SI) is approximately parallel to 

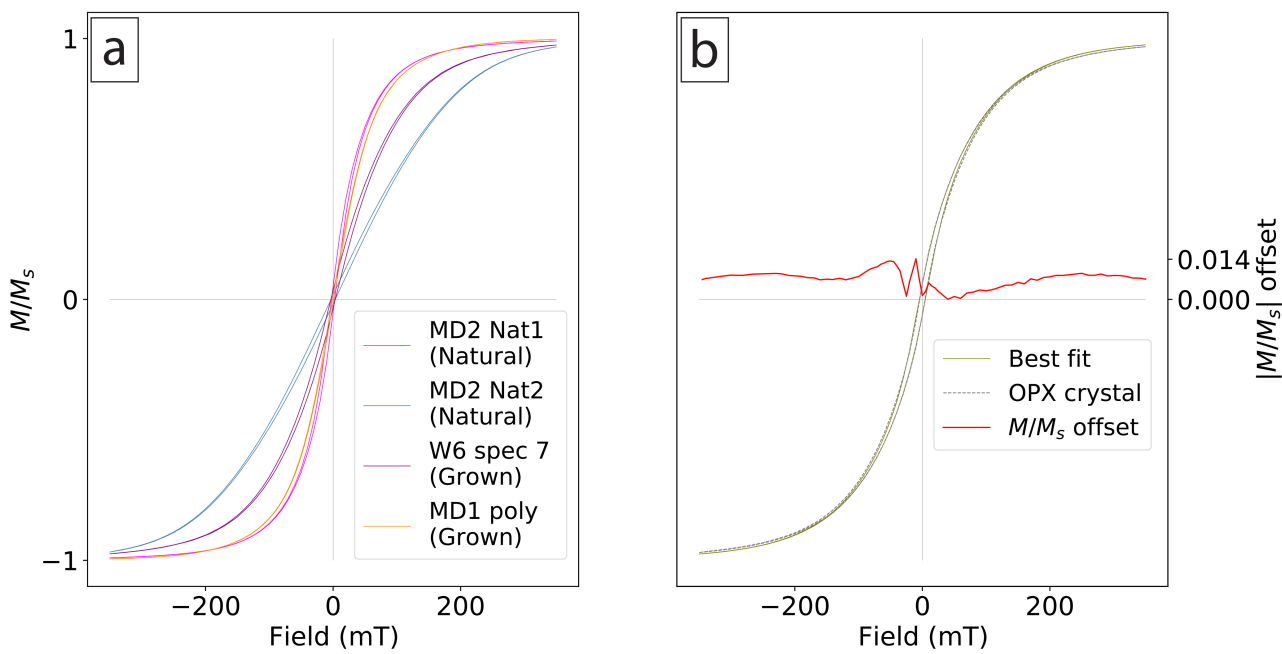

Figure 7. (a) Magnetic hysteresis curves for fields from -350 to $350 \mathrm{mT}$ of natural and grown magnetite with multi domain magnetic parameters that have no influence of single domain material. (b) The resulting composite curve by non-negative least-square optimization compared to the measured single crystal of orthopyroxene. $H_{c}$ and $M_{r s} / M_{s}$ is presented in Tab. 4

the $[010]_{\mathrm{opx}}$ in external field direction 8 . The minimum $\chi^{*}$ with 2.7 (SI) is sub parallel to $[100]_{\text {opx }}$ in field directions 15 and 18, which are the same directions as the lowest $M_{r s} / M_{s}$.

In the plagioclase, the highest $M_{r s} / M_{s}$ values of $0.510-0.518$ in Tab. A.2 aligns with the fitted $[\overline{1} \overline{2} 8]_{\text {plag }}$ direction and in the external field direction 12 and 15 . Which is highly influenced by the primary $[001]_{\text {plag }}$ cluster of oriented magnetite, but offset by the contribution of the lesser magnetite populated normal to the $(150)_{\text {plag }}$ plane. The lowest $M_{r s} / M_{s}$ at 0.198 oriented at $\phi=75^{\circ}$ to the maximum $M_{r s} / M_{s}$ in the external field direction 8. The maximum $H_{c}$ of $46 \mathrm{mT}$ is in direction 11 and the minimum of $22 \mathrm{mT}$ in direction 13. For $\chi^{*}$ the maximum of $5.8(\mathrm{SI})$ is in the external field direction 9 and the minimum of 3.3 (SI) in the external field direction 16. These results indicate that for plagioclase the lowest $M_{r s} / M_{s}$ and $\chi^{*}$ are in the same direction as maximum $H_{c}$.

Tab. 3 summarizes the micromagnetic properties for the hysteresis curves and the extracted silicate volumes providing an estimate for the magnetic properties as they are measured on the bulk sample. The first and third row represents the measured single-crystal hysteresis and second and fourth row the milled volumes. Volume $(V)$ is calculated from the weight of the silicate crystal with an average mineral density and the MERRILL volume is estimated by the size of the FIB cube (Fig. 3 and 2). The saturated moment $\left(\mu_{s}\right), M_{r s} / M_{s}$ and $H_{c}$ 
are interpreted from the hysteresis. Saturation magnetization $\left(M_{S}\right)$ is calculated $\mu_{S} / V$ and represents the magnetic intensity of the magnetite inclusions.

\section{Discussion}

\subsection{Orthopyroxene hysteresis}

A comparison of the measured hysteresis loop of the orthopyroxene single crystals with the MERRILL calculated hysteresis loop in Fig. 6 highlights the limitations of this approach. Based on optical observations, the most abundant magnetite occurs in two groups of $500-300 \mu \mathrm{m}$ and $50-100 \mu \mathrm{m}$ oxy-exsolved inclusions of MD magnetite and ilmenite. The area targeted for MERRILL calculations, on the other hand, focused on the rarer occurrence of much smaller magnetite lamellae (Fig. A.4 and A.2c). Of the 68 particles within the milled orthopyroxene volume (Fig. 3), the largest particles that were completed in MERRILL have a volume equivalent to a sphere diameter (EVSD) of $<400 \mathrm{~nm}$ and adopt MV states. The median $H_{c}$ and $M_{r s} / M_{s}$ values of these particles are $0.02 \mathrm{~T}$ and 0.51 , respectively - leading to considerably higher coercivity and remanence in the calculated loop compared to the measured one. The best hysteresis curve fit in Fig. $6 \mathrm{~b}$ implies that $\approx 94 \%$ of the magnetic signal is due to MD magnetite (Fig. $6 \mathrm{~b}$ and Tab. 4. The OPX $M_{s}$ values in Tab. 3 provide the estimate that OPX MERRILL represents $177 / 2234 \approx 8 \%$ of the bulk OPX singlecrystal measurement. Together, this defines a calculated range of the MD contribution to the measured single-crystal hysteresis for this particular sample between $92-94 \%$. Even though the MERRILL curve contributes only $6 \%$ to the total saturation magnetisation of the sample, its SD-MV particles carry a disproportionately large $28 \%$ of the remanence in these silicates (Tab. 4).

Optical and electron microscopy observations indicate that thin magnetite lamellae which correspond to the model volume in Fig. 3 are scarcer than $6 \%$ and occur in patches with associated In-Pig lamellae (Fig. A.4 and A.3). However, there are also grain boundary and crack-filling Fe-oxides (Fig. A.3, A.2a, A.2b and A.2e). These particles are not identified properly, but would contribute to the measured hysteresis and could therefore explain the minor differences between the optical estimate $(<6 \%)$ and that based on the magnetic properties (6-8\%). An overall bulk estimate of magnetite based on optical microscopy observations puts the MD fraction at $97-98 \%$. 
Table 4. Magnetic properties $M_{r s} / M_{s}$ and $H_{c}(\mathrm{mT})$ for the MD hysteresis curves and the MERRILL calculated hysteresis curve. Weight percent $(\mathrm{Wt} \%)$ and the remanence fraction $(\mathrm{RF}(\%))$ is the contribution of each hysteresis curve to the calculated best fit curve.

\begin{tabular}{cccccc} 
Hysteresis & Mass $(\mathrm{g})$ & $M_{r s} / M_{s}$ & $H_{c}(\mathrm{mT})$ & $\mathrm{Wt} \%$ & $\mathrm{RF}(\%)$ \\
\hline MD1 poly & $5.7 \times 10^{-4}$ & 0.01 & 0.723 & 0 & 0 \\
MD2 nat1 & $1.7 \times 10^{-1}$ & 0.05 & 2.5 & 29.4 & 17.2 \\
MD2 nat2 & $1.7 \times 10^{-1}$ & 0.017 & 3.135 & 16.7 & 12.6 \\
W6 spec 7 & $4.9 \times 10^{-3}$ & 0.033 & 3.35 & 48.3 & 41.7 \\
& & & & & \\
MERRILL & $7.23 \times 10^{-10}$ & 0.46 & 21.88 & 5.6 & 28.4
\end{tabular}

\subsection{Plagioclase hysteresis}

For plagioclase in Tab. 3 the $M_{S}$ for measured hysteresis is $51.6(\mathrm{~A} / \mathrm{m})$ compared with 93.8 (A/m) for the MERRILL calculations. This nearly two-fold greater magnetization of the milled volume can be explained by selective sampling. Since the limitation for the milling volume is about $25 \mu \mathrm{m} \times 25 \mu \mathrm{m} \times 15 \mu \mathrm{m}(\mathrm{L} \times \mathrm{W} \times \mathrm{H})$ it is important to select an area with a high concentration of visible inclusions. It is, therefore, highly probable that the area selection is biased towards a higher concentration of larger particles, since it is difficult to observe and evaluate the concentration of smaller inclusions that are suitable for modelling in MERRILL. The much higher $H_{c}$ of the measured hysteresis loop (Fig.6b) is consistent with the idea that, on average, particles contained in the feldspar crystals are smaller (more SD) than those represented in the region selected for FIB milling. The modelled particles with $H_{c}$ in the range of $65 \mathrm{mT}$ are predominantly SD particles with $M_{r s} / M_{s} \approx 0.5$. The particles with $M_{r s} / M_{s}$ in the range of 0.4 are primarily SV to MV particles with $5 \mathrm{mT}<H_{c}<40 \mathrm{mT}$. The difference in $H_{c}$ values of $37 \mathrm{mT}$ (model) and $65 \mathrm{mT}$ can also be due to the effect of dislocations, surface stresses and pinning sites. These natural imperfections delay the magnetization change and increase $H_{c}$, and are not included in the micromagnetic model, where by the latter underestimates $H_{c}$.

These observations demonstrate the importance of recognising the sampling bias that is inherent with a highly targeted approach such as FIB micromagnetic nanotomography, which self-selects particles in the SD-MV size range (limited at small sizes by the finite 
thickness of the slices and at large sizes by the computational constraints of the micromagnetic simulations). The fact that observations are destructive, and contained within a limited fieldof-view that may poorly represent the particle size distribution in the whole crystal, must also be considered. Techniques such as high-resolution X-ray tomography, which are capable of non-destructive imaging of entire single crystals [Suuronen and Sayab, 2018] have the potential to reduce sampling bias associated with the limited field of view. However, such methods are currently only able to detect particles in the SV range and above due to a combination of contrast and spatial resolution limitations. A multi-scale, correlative approach, that combines high-resolution X-ray tomography of the whole crystal with targeted FIB nanotomography and transmission electron microscopy, would enable full characterisation across the entire range SP-SD-SV-MV-MD domain states. Pushing micromagnetic simulations into the MD range, however, remains a significant computational challenge.

\subsection{Magnetic anisotropy of $M_{r s} / M_{s}, H_{c}$, susceptibility and the deflection of remanent magnetization}

The following subsections discuss and analyze the modeled average anisotropy over all particles for three parameters based on the 20 modeled field directions. These parameters are not completely independent, but focus on different aspects of the magnetic ensemble. $M_{r s} / M_{s}$ indicates the strength of remanence, $H_{c}$ the stability of remanence and $\chi^{*}$ the overall ease of field response of the magnetization structures. Because these are average results for individual field directions, the values for individual particles can vary significantly. As the different sections of the discussion do cross reference and depend on each other, we have compiled the following fig. 8, 10 and 9 into the single supplementary Fig. A.6.

\subsubsection{Anisotropy of $M_{r s} / M_{S}$ and $H_{c}$}

The field orientation dependence of ensemble hysteresis properties is illustrated in Fig. 8 using the colour and size of the symbols to indicate $M_{r s} / M_{s}$ and $H_{c}$ from Tab. A.1, respectively. The crystallographic orientations of the pyroxene and feldspar single crystals, as determined by EBSD are indicated, along with reference crystallographic directions and planes. Contours illustrate the distribution of elongation axes of the magnetite particles, as determined by FIB. In the orthopyroxene (Fig. 8a) the primary cluster of elongation axes is oriented along $[001]_{\mathrm{opx}}$ and a smaller cluster elongated along $[010]_{\mathrm{opx}}$. Whilst the maximum $M_{r s} / M_{s}$ trend corresponds well to that expected based on shape anisotropy, the $H_{c}$ trend 
is arguably less intuitive, with highest $H_{c}$ in the direction of the lowest $M_{r s} / M_{s}$. Because the particle distribution is dominated by SD and SV domain states, the $M_{r s} / M_{s}$ and $H_{c}$ anisotropy should be different from the anisotropy expected for Stoner-Wohlfarth SD hysteresis. The shape of the modeled OPX MERRILL hysteresis loop (Fig. 6a) would usually be classified as SD dominated even though it is not. Because the calculated magnetic anisotropy is different from the Stoner-Wohlfarth model, it is likely dominated by the non-SD states in the modeled particle ensemble.

Magnetic models in Fig. 4 represent typical particles that adopt SD, SV or MV remanent states after energy minimisation from a random seed, but display different remanent states after exposure to a saturating field. For example, crystal OPX026 is an approximately $1 \mu \mathrm{m}$ long needle that adopts an SD state after random minimisation (left image in Fig. 4) and after exposure to a saturating field applied close to its elongation direction (right and central image in Fig. 4). The other crystals in Fig. 4 adopt SV or MV states after random minimisation, but a range of more SD-like buckling states [e.g. Aharoni, 2000] after exposure to saturating fields in different directions. Fig. 4 demonstrates that the maximum $M_{r s} / M_{s}$ occurs for fields applied along the long axis, and the minimum $M_{r s} / M_{s}$ for fields along the short axis. When the external field is oriented at a high angle to the elongation axis, the switching takes place by a different succession of magnetization structures. An SD flower state at $0 \mathrm{mT}$ rapidly nucleates an oscillatory buckling configuration with a shorter wavelength. This state is now relatively stable while the magnetization slowly rotates towards the external field direction. At higher opposite field strength the oscillatory buckling state nucleates a vortex. In the case of OPX071, the maximum coercivity $H_{c}$ is observed for fields applied at a high angle to the elongation direction, where the adopted oscillatory buckling state in Fig. 4 (OPX071,\#F:15) can be reversibly changed between $0-35 \mathrm{mT}$. At higher absolute fields the magnetization structure switches irreversibly and finally nucleates a single vortex. With external fields oriented sub-parallel to the particle's elongation axis the domain structure at zero field is reminiscent of a SD flower state. Increasing the field in opposite direction generates oscillatory buckling states [DeSimone et al., 2005] with long wavelengths which eventually nucleate to a single vortex. Further field increase propagates the vortex core towards the particle edge and after de-nucleation transforms to a field-aligned flower or SD state.

In the plagioclase (Fig. 8b) the anisotropy of hysteresis parameters is weaker than in pyroxene. Contours indicate three distinct populations of particles with the most populated in the $[001]_{\text {plag }}$ orientation and the lesser populated normal to the $(150)_{\text {plag }}$ plane and the 


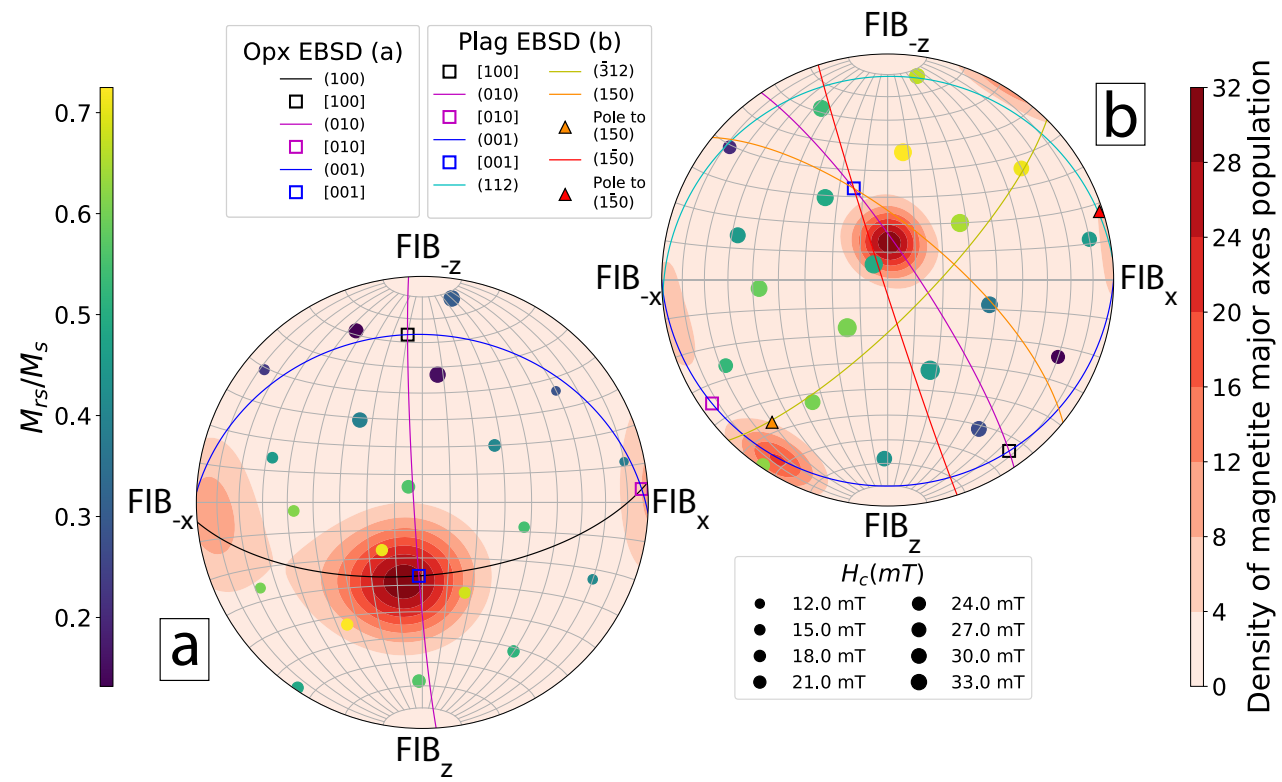

Figure 8. Stereographic projection normal to the $F I B_{Y}$-axis for both orthopyroxene (Fig. 3a) and plagioclase (Fig. 2b). Red contours show the population of the elongation axis for the particles and the round markers the external field direction. Round marker size is the total coercivity and color is the strength of the remanence. Lines show the crystallographic planes of the silicate host and the square markers the crystallographic direction. 
normal to the $(1 \overline{5} 0)_{\text {plag }}$ plane. The highest $M_{r s} / M_{s}$ values (yellows and greens in Fig. $8 \mathrm{~b}$ ) are observed in a plane defined by the primary and secondary clusters of magnetite elongation axes, where the lower $M_{r s} / M_{s}$ is oriented in the direction of the pole for this plane. The absolute maximum $M_{r s} / M_{s}$ aligns with the fitted [ $\left.\overline{1} \overline{2} 8\right]_{\text {plag }}$ direction, which is highly influenced by the primary $[001]_{\text {plag }}$ cluster of oriented magnetite, but offset by the contribution of the lesser magnetite populated normal to the (150) plag plane. For $H_{c}$ the highest values ( $\geq 39 \mathrm{mT}$ ) occur where external field directions are oriented close to parallel with the $[001]_{\text {plag }}$ magnetite population. The lowest $H_{c}$ is quite distinct and occurs where the external field is at the highest angles to the main magnetite population and their elongation directions. In contrast to the orthopyroxene, the direction of maximum $M_{r s} / M_{s}$ and $H_{c}$ is close to the elongation direction of magnetite. A correlation that is theoretically expected, based on the Stoner-Wohlfarth model of SD hysteresis.

\subsubsection{Deflection of remanent magnetization}

The physical origin of the anisotropy of remanent magnetization are deflections of the individual grains towards collective preferred directions. In Fig. 9 this effect is visualized by showing the averages of the deflected remanence directions (red marks) for all modeled field directions. The direction of the remanence vectors for magnetic particles in orthopyroxene (Fig. 9a) are consistent with the field directions with the highest $M_{r s} / M_{s}$ in Fig. 8a, highlighting the strong ferrimagnetic fabric in OPX aligned with the magnetite elongation axis. Two of the 20 remanence vectors in Fig. 9a form a group outside the main clusters. They belong to the external field directions 2 and 7 (Table A.1 and Fig. A.1), and are oriented in the [010] $]_{\mathrm{px}}$ direction. The group is separate, because it is populated by a relatively large fraction of particles with elongation axes along the [010] $]_{\text {opx }}$ (Fig. 8a), like OPX071 in Fig. 4. They can also lock a high remanent magnetization in this direction. For plagioclase, the directions in which the remenanence is deflected in Fig. $9 \mathrm{~b}$ differ from the orientations of the $M_{r s} / M_{s}$ and $H_{c}$ in Fig. 8b. The main direction of deflection of $M_{r s} / M_{s}$ is in the [010] plag and almost perpendicular to the direction of maximal $M_{r s} / M_{s}$. Maximum $H_{c}$ and $M_{r s} / M_{s}$ are oriented in the $[001]_{\text {plag }}$ direction, but both also show an alignment with elevated intensities in the best fit plane for the remanence in Fig. 9b. This behavior is due to the fact, that in the best fit plane the exact direction of deflection or $M_{r s} / M_{s}$ is relatively indetermined, because the two primary populations of elongation axes contribute in nearly equal amounts. 

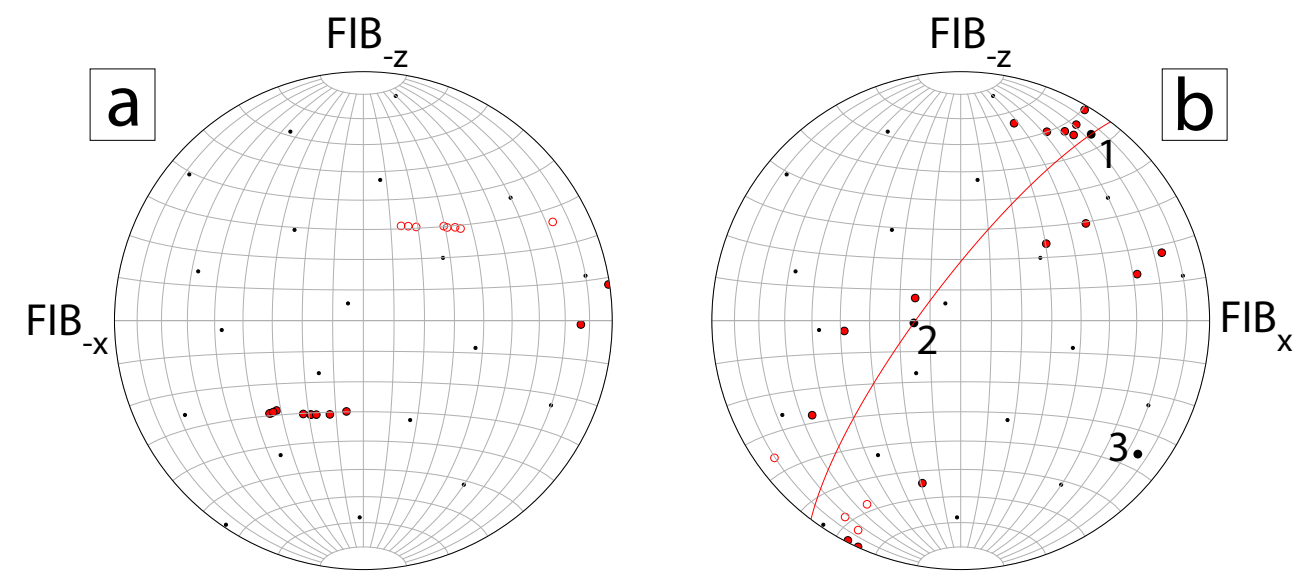

- Best fit plane for remanence vectors
External field directions (Fig. A.1)
Remanence direction in the upper hemisphere (Tab. A.1)
Remanence direction in the lower hemisphere (Tab. A.1)
Remanence eigenvector directions

Figure 9. Deflected remanence vectors (red) for all modeled field directions (small black dots) after averaging over the particle ensemble. Red disks (circles) mark directions in the lower (upper) hemisphere (see Table A.1). a) Results for for orthopyroxene (OPX). b) Results for plagioclase (PLAG). The red great circle represents the best fit plane for the deflected remanence vectors. Eigenvector directions of the remanent magnetizations are indicated by large numbered black disks. These deflections are the physical origin of the anisotropy of remanent magnetization. 


\subsubsection{Anisotropy of magnetic susceptibility in the remanent state}

The average absolute values of $\chi^{*}$ for both silicates lie in the range of $3-5$ (SI), which coincides with Figure 1 in Heider et al. [1996], where experimentally determined isotropic initial magnetic susceptibilities $\left(\chi_{0}\right)$ of magnetite fall in the range of $2-4$ (SI). For pyroxene our $\chi^{*}$ reach values of $10-15(\mathrm{SI})$ for field directions at low angle to the particles elongation axis indicating a substantial anisotropy. The ensemble magnetic susceptibility tensor (k) for all pyroxene particles, where $\mathbf{k}_{1}>\mathbf{k}_{2} \gg \mathbf{k}_{3}$ describes an oblate anisotropy, where $\mathbf{k}_{1}$ is sub-parallel to the $[001]_{\text {opx }}, \mathbf{k}_{2}$ sub-parallel to the $[010]_{\text {opx }}$ direction and $\mathbf{k}_{3}$ sub-parallel to the $[100]_{\text {opx }}$ direction (Fig. 10a). The $\mathbf{k}_{1}$ direction is correlated with the primary cluster of individual particle elongation axes, which is parallel to the maximum $M_{r s} / M_{s}$ (Fig. 8a). The large ensemble value of $\mathbf{k}_{2}$ is related to the secondary peak of individual particle elongation directions in Fig. 8a. The ensemble value of $\mathbf{k}_{3}$ is substantially smaller because no individual particle elongations are close to the corresponding direction of $[100]_{\mathrm{opx}}$.

The micromagnetic models allow us to analyze which changes in the magnetization structure are responsible for the calculated susceptibilities. This is done by comparing the magnetization state $\mathbf{m}_{1}$ at $5 \mathrm{mT}$ to the state $\mathbf{m}_{2}$ at $-5 \mathrm{mT}$. If the lengths of the difference magnetization vectors $\Delta m=\left|\mathbf{m}_{1}-\mathbf{m}_{2}\right|$ at all mesh nodes are similar, a global rotation of spins has occurred. If for some nodes $\Delta m$ is much larger than for others, a localized shift of magnetization took place near the locations of higher $\Delta m$. For particle OPX071 with fields at a low angle to the elongation axis, inspecting the magnetization change shows that localized changes with high $\Delta m$ occur via increased magnetization buckling. The same particle with a field at high angles displays much smaller global $\Delta m$, indicating a stiff magnetization structure which allows only tiny rotations in response to an external field.

The review by Biedermann [2018] investigates low field anisotropy of magnetic susceptibility (AMS) carried by silicates and shows that measuring the principal components of $k$ in pyroxene single crystals can provide a wide range of results. Biedermann [2018] reviews the few studies that have conducted research on this subject and suggests that inconsistencies result from ferromagnetic inclusions or exsolved magnetic phases within the silicate. For these studies the AMS of exsolved phases can contribute to, or even dominate, the intrinsic paramagnetic magnetic anisotropy of the silicate crystal itself. Methods to separate paramagnetic and ferromagnetic anisotropy are purely based on bulk measurements [Martín-Hernández and Ferré, 2007; Richter and van der Pluijm, 1994]. Since our study removes the silicate host all 


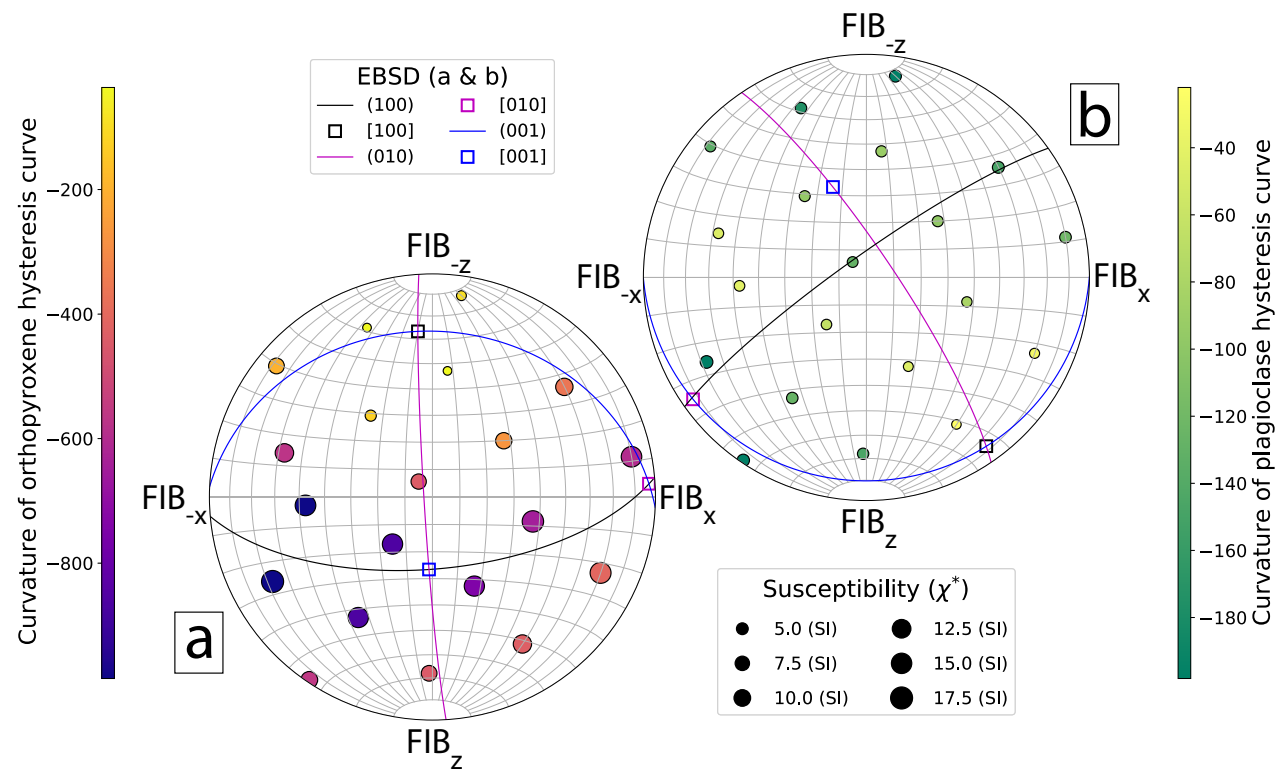

Figure 10. Stereographic projection down the y-axis for the milled volume in both (a) orthopyroxene

(Fig. 3) and (b) plagioclase (Fig. 2). Round marker size is the calculated susceptebility ( $\left.\chi^{*}\right)$ in the direction of the external field. Color is the curvature of the upper hysteresis branch at $0 \mathrm{mT}$ in the direction of the external field.

together, there is no potential paramagnetic contribution, and our results can help to define the pure ferromagnetic contribution to the total AMS. Biedermann [2018] states that the degree of anisotropy increases with increasing amount of iron and the shape of the anisotropy changes from prolate to oblate, without providing any indication to where the additional $\mathrm{Fe}$ is situated. Their observation, combined with our results, where $k_{1}>k_{2} \gg k_{3}$, is consistent with the hypothesis that additional iron increases the ferromagnetic contribution to the AMS, by the formation of exsolved magnetite with an oblate anisotropy.

AMS in plagioclase single crystals is weak and often dominated by a diamagnetic component [Biedermann et al., 2020]. Our results in Fig. 10b do reflect a lower ferromagnetic anisotropy than for pyroxene, with $k_{1}$ in the direction of $[010]_{\text {plag }}, k_{2}$ parallel to the particle elongation axis population slightly canted to the [001 $]_{\text {plag }}$ direction (Fig. 8) and $k_{3}$ parallel to the $[100]_{\text {plag }}$ direction. The direction of the highest $\chi^{*}$ is oriented sub-parallel to the particle populations elongated normal to $(150)_{\text {plag }}$ and $(\overline{150})_{\text {plag }}$, rather than in the direction of maximum $M_{r s} / M_{s}$ as seen for the orthopyroxene. A reason is the way we calculated $\chi^{*}$ as the first derivative of the hysteresis curve at $0 \mathrm{mT}$. The calculated orthopyroxene curves (Fig. 5a) 
with the highest $M_{r s} / M_{s}$ all have a steep gradient, but for the plagioclase curves (Fig. $5 \mathrm{~b}$ ) the gradients are all very similar. $\chi^{*}$ can be misleading because the magnetic models were minimized from a saturating field strength and the hysteresis resolution (field increments) is limited. However, the direction of maximum $\chi^{*}$ is supported by measurements of sunstone (andesine) single-crystal. Measurements by Hirt and Biedermann [2019] show $k_{1}$ in the direction of $[010]_{\text {plag }}$ and documented to be along the lath direction of ferrimangentic exolutions. Other measurements that include labradorite concluded the high-field AMS to be very weak, but showing the $k_{1}$ in the [010] plag and $k_{3}$ in [001 $]_{\text {plag }}$ directions [Hirt and Biedermann, 2019; Biedermann et al., 2016].

Since we have chosen $5 \mathrm{mT}$ steps for the external field there are details about the changes to the domain state at remanence that are not showing up in our simulations. Here further calculations with denser field steps and more field directions would be needed to provide a better understanding of these observations. The combination of FIB nanotomography and micromagnetic simulations in this case plays a valuable role in defining what our expectations should be for the intrinsic anisotropy of silicate-hosted magnetic inclusions.

\subsection{Outlook}

Micromagnetic modelling can provide valuable insights when interpreting bulk magnetic properties. Our comparison between single crystal measurements and the summarized MERRILL calculations highlight the magnetic diversity when analyzing individual components at different scales. For plagioclase the measured and calculated hysteresis have a good overlap, but unexpectedly the measured hysteresis has a higher coercivity that our model was not able to replicate. One of reasons may be a selection bias of the FIB volume, but also unmodeled influences on coercivity are possible. Our results show that for plagioclase there is only a slight difference in the magnetic properties when analysing substantially different volumes. For the pyroxene our study highlights exactly how complicated the analysis of such silicates can become. This complexity is demonstrated by the disagreement between the measured and calculated hysteresis loops, that separately indicate there will be a deviation in the magentic properties when analysing different volumes. From our calculations the directions of remanence, susceptibility, high $H_{c}$ and high $M_{r s} / M_{s}$ are strongly oriented in specific crystallographic orientations. These important results, even at the scale of single-crystal measurements can be masked by secondary magnetic particles, magnetic inclusions and magnetic exsolutions in coexisting silicates, like quartz inclusions in plagioclase and inverted pigionite in the orthopyroxene. 
These are challenges that rock magnetism has to overcome and the results from Nikolaisen et al. [2020] and this study, provide a pathway to tackle this monumental task. Our models/calculations identify the primary oxide carriers of stable remanence, their geometries and how these are situated within the host and most importantly their micromagentic properties, thereby illustrating the importance of further studies that combine FIB nanotomography and micromagnetic simulations.

\section{Conclusion}

This study shows the possibility of studying primary remanence carriers from first principles by finite-element micromagnetic modelling. By individually modeling 302 silicatehosted magnetite particles and comparing with single-crystal hysteresis measurements, we are able to show collectively and individually the source of stable remanence within these samples.

In orthopyroxene, magnetite exsolves as oblate particles elongated sub-parallel to the $[001]_{\text {opx }}$ direction. The summarized hysteresis have a dominant SD response that strongly contrasts with the single-crystal MD dominated hysteresis. By adding weighted fractions of MD hysteresis to the magnetic modelling, we managed to replicate the measured hysteresis with $1.6 \%$ error. The MERRILL-calculated micromagnetics have contributed just $6 \%$ to the hysteresis loop, but contributes $28 \%$ to the remanent magnetization. The maximum $M_{r s} / M_{s}$, calculated magnetic susceptibility $\left(\chi^{*}\right)$ and the remanence direction is strongly correlated to elongation axes of magnetite and therefore the $[001]_{\mathrm{opx}}$ direction. The maximum $H_{c}$ is oriented in the $[100]_{\mathrm{opx}}$ and parallel to the minimum $\chi^{*}$, which reflects larger vortex nucleation fields when the applied field direction approaches the short axis. The maximum $H_{c}$ is therefore orthogonal to the maximum $M_{r s} / M_{s}$, which is controlled by axis-aligned metastable singledomain states at zero field.

In plagioclase the magnetite exsolves as elongated needles that are primarily oriented in the $[001]_{\text {plag }}$ direction and those normal to the $(150)_{\text {plag }}$ and $(1 \overline{5} 0)_{\text {plag }}$ planes. The direction of magnetic remanence is in the direction of the magnetite population normal to the $(150)_{\text {plag }}$ and $k_{1}$ in the $[010]_{\text {plag }}$ direction. The maximum $M_{r s} / M_{s}$ and maximum $H_{c}$ align sub-parallel to the $[001]_{\text {plag }}$ indicating the primary remanence carriers are SD. The $M_{r s} / M_{s}$ values of measured (0.4) and modelled (0.396) hysteresis curves differ only by $1 \%$, which we interpret to show that the remanent magnetization of micromagnetic models for plagioclase accurately 
represent the equililibrium magnetization of the bulk single crystal at its saturation remanence state. The surprisingly high $H_{c}$ of the measured hysteresis loop can result from a variety of factors. The limited FIB interaction volume and pinning by natural imperfections, not included in the micromagnetic modelling, could explain the observed difference between the modelled $(37 \mathrm{mT})$ and measured $(65 \mathrm{mT})$ hysteresis loop.

These results provide the first statistically robust connection between micromagnetically modeled natural particle ensembles and bulk hysteresis measurements from the same samples. Small magnetite grain sizes are proportionally more important for remanent magnetization than for induced magnetization, and provide quantitative estimates of the two samples where FIB data are available. Our results, based on FIB, EBSD, and MERRILL modeling indicate that the orthogonality relationship between the directions of maximal $M_{r s} / M_{s}$ and maximal $H_{c}$ may serve as an experimental indicator for the dominance of metastable SD particles as carriers of the remanent magnetization in single host crystals. The AMS of presumably paramagnetic single silicate crystals correlate suspiciously well with our calculations. This raises the question if the intended removal of ferromagnetic susceptibility from the measurement was really successful, even though a lattice aligned precipitation of ferrimagnetic particles could also by chance mimic the paramagnetic AMS. This study is the first of its kind, and it is important that future studies expand on these results to include a variety of silicates (micas, amphiboles, pyroxenes and plagioclase) and oxides (magnetite, ilmentite, ulvöspinel and hematite), thereby contributing to the understanding of magnetic particles from a fundamental perspective to the total magnetic hysteresis assemblage.

\section{Acknowledgments}

The FIB-SEM and Slice-and-View work was preformed at the NTNU Nanolab and the Norwegian infrastructure for micro- and nanofabrication as part of a PhD-project funded by NTNU. The Research Council of Norway is acknowledged for the support to the Norwegian Micro-and Nano-Fabrication Facility, NorFab, Project 245963/F50. The calculations and usage of a Linux server was possible with granted access by IGP and a designated workstation with remote access. The authors would like to thank Annemarieke Béguin for supplying measured MD hysteresis of the W6 spec 7 sample. We wish to thank the two anonymous reviewers for their thorough and constructive reviews.

Data Availability Statement The summarized magnetic properties and individual particles shapes used in this article are available in [dataverse], at https://doi.org/10.18710/AZT6UY 
[doi]. Further detailed micromagnetic simulations of individual grains in each external filed directions are available upon request, they are not in the database because of the substantial collective file sizes.

Author contribution statement Even Nikolaisen (E N), Richard Harrison (R H) and Karl Fabian (K F) conceived the idea of this manuscript. E N performed computations, processing and analysis of data and created the figures. EN wrote the draft of the manuscript, then R H, K F and Suzanne McEnroe contributed suggestions and edits to the discussion and conclusions. Nathan Church wrote the method section of the bulk magnetic measurements and provided the single crystal hysteresis data. Bjørn Eske Sørensen wrote the EBSD method section and performed the EBSD measurements and analysis. Christian Tegner provided the chemical analysis, the sample, and edited the material presentation.

\section{References}

Ageeva, O., G. Bian, G. Habler, A. Pertsev, and R. Abart (2020), Crystallographic and shape orientations of magnetite micro-inclusions in plagioclase, Contributions to Mineralogy and Petrology, 175(10), 1-16.

Aharoni, A. (2000), Introduction to the Theory of Ferromagnetism, 2nd ed., Oxford University Press, Oxford.

Ayachit, U. (2015), The paraview guide: a parallel visualization application, Kitware, Inc.

Bachmann, F., R. Hielscher, and H. Schaeben (2010), Texture analysis with mtex-free and open source software toolbox, Solid State Phenomena, 160, 63-68.

Biedermann, A. R. (2018), Magnetic anisotropy in single crystals: A review, Geosciences, 8(8), 302.

Biedermann, A. R., T. Pettke, R. J. Angel, and A. M. Hirt (2016), Anisotropy of magnetic susceptibility in alkali feldspar and plagioclase, Geophysical Supplements to the Monthly Notices of the Royal Astronomical Society, 205(1), 479-489.

Biedermann, A. R., M. Jackson, M. Chadima, A. M. Hirt, and J. M. Feinberg (2020), Beyond the second-order magnetic anisotropy tensor: higher-order components due to oriented magnetite exsolutions in pyroxenes, and implications for palaeomagnetic and structural interpretations, Geophysical journal international, 223(2), 915-933.

Bono, R. K., J. A. Tarduno, F. Nimmo, and R. D. Cottrell (2019), Young inner core inferred from ediacaran ultra-low geomagnetic field intensity, Nature Geoscience, 12(2), 143-147. 
Buades, A., B. Coll, and J.-M. Morel (2011), Non-local means denoising, Image Processing On Line, 1, 208-212.

Burton-Johnson, A., C. Macpherson, J. Muraszko, R. Harrison, and T. A. Jordan (2019), Tectonic strain recorded by magnetic fabrics (ams) in plutons, including mt kinabalu, borneo: A tool to explore past tectonic regimes and syn-magmatic deformation, Journal of Structural Geology, 119, 50-60.

Cawthorn, R., G. Cooper, and S. Webb (1998), Connectivity between the western and eastern limbs of the bushveld complex, South African Journal of Geology, 101(4), 291-298.

Cawthorn, R. G., K. L. Lundgaard, C. Tegner, and J. R. Wilson (2016), Lateral variations in plagioclase compositions, main zone, bushveld complex, south africa: Evidence for slow mixing of magmas in basinal structures, Mineralogical Magazine, 80(2), 213-225.

Champness, P., and G. Lorimer (1974), A direct lattice-resolution study of precipitation (exsolution) in orthopyroxene, Philosophical Magazine, 30(2), 357-365.

Church, N. S., J. M. Feinberg, and R. J. Harrison (2011), Low-temperature domain wall pinning in titanomagnetite: Quantitative modeling of multidomain first-order reversal curve diagrams and AC susceptibility, Geochemistry Geophysics Geosystems, 12(7), Q07Z27, doi:10.1029/2011GC003538.

Crowson, P. (2001), Minerals Handbook 2000-01: Statistics \& Analyses of the World's Minerals Industry, Mining journal books.

Darbon, J., A. Cunha, T. F. Chan, S. Osher, and G. J. Jensen (2008), Fast nonlocal filtering applied to electron cryomicroscopy, in 2008 5th IEEE International Symposium on biomedical imaging: from nano to macro, pp. 1331-1334, IEEE.

Davis, K. E. (1981), Magnetite rods in plagioclase as the primary carrier of stable nrm in ocean floor gabbros, Earth and Planetary Science Letters, 55(1), 190-198.

Dawson-Haggerty et al. (2019), trimesh.

DeSimone, A., R. V. Kohn, S. Müller, and F. Otto (2005), Recent analytical developments in micromagnetics, in The Science of Hysteresis, vol. II, edited by G. Bertotti and I. Mayergoyz, chap. 4, pp. 269-381, Elsevier.

Dieckmann, R. (1982), Defects and Cation Diffusion in Magnetite (IV): Nonstoichiometry and Point Defect Structure of Magnetite $\left(\mathrm{Fe}_{3-\delta} \mathrm{O}_{4}\right)$, Berichte der Bunsengesellschaft für physikalische Chemie, 86(2), 112-118, doi:10.1002/bbpc.19820860205.

Fabian, K., and V. P. Shcherbakov (2018), Energy barriers in three-dimensional micromagnetic models and the physics of thermoviscous magnetization, Geophysical 
Journal International, 215(1), 314-324, doi:10.1093/gji/ggy285.

Feinberg, J. M., H.-R. Wenk, P. R. Renne, and G. R. Scott (2004), Epitaxial relationships of clinopyroxene-hosted magnetite determined using electron backscatter diffraction (ebsd) technique, American Mineralogist, 89(2-3), 462-466.

Feinberg, J. M., G. R. Scott, P. R. Renne, and H.-R. Wenk (2005), Exsolved magnetite inclusions in silicates: Features determining their remanence behavior, Geology, 33(6), $513-516$.

Feinberg, J. M., H.-R. Wenk, G. R. Scott, and P. R. Renne (2006), Preferred orientation and anisotropy of seismic and magnetic properties in gabbronorites from the bushveld layered intrusion, Tectonophysics, 420(3-4), 345-356.

Fleet, M., G. A. Bilcox, and R. L. Barnett (1980), Oriented magnetite inclusions in pyroxenes from the grenville province, The Canadian Mineralogist, 18(1), 89-99.

Frandsen, C., S. Stipp, S. McEnroe, M. Madsen, and J. Knudsen (2004), Magnetic domain structures and stray fields of individual elongated magnetite grains revealed by magnetic force microscopy (mfm), Physics of the Earth and Planetary Interiors, 141(2), 121-129.

Hattingh, P. (1986), The palaeomagnetism of the main zone of the eastern bushveld complex, Tectonophysics, 124(3-4), 271-295.

Heider, F., A. Zitzelsberger, and K. Fabian (1996), Magnetic susceptibility and remanent coercive force in grown magnetite crystals from $0.1 \mu \mathrm{m}$ to $6 \mathrm{~mm}$, Physics of the Earth and Planetary interiors, 93(3-4), 239-256.

Hirt, A. M., and A. R. Biedermann (2019), Preferred orientation of ferromagnetic phases in rock-forming minerals: insights from magnetic anisotropy of single crystals, Canadian journal of earth sciences, 56(9), 994-1001.

Kirby, S., and M. Etheridge (1981), Exsolution of ca-clinopyroxene from orthopyroxene aided by deformation, Physics and Chemistry of Minerals, 7(3), 105-109.

Krasa, D., C. Heunemann, R. Leonhardt, and N. Petersen (2003), Experimental procedure to detect multidomain remanence during Thellier-Thellier experiments, Physics and Chemistry of the Earth, 28(16-19), 681-687, doi:10.1016/S1474-7065(03)00122-0.

Lawson, C. L., and R. J. Hanson (1995), Solving least squares problems, SIAM.

Letts, S., T. H. Torsvik, S. J. Webb, and L. D. Ashwal (2009), Palaeomagnetism of the 2054 ma bushveld complex (south africa): implications for emplacement and cooling, Geophysical Journal International, 179(2), 850-872. 
Lundgaard, K. L. (2003), Magma Chamber Processes Studied in Two Layered Instrusions [including 4 Papers], Aarhus University, Department of Earth Sciences.

Lundgaard, K. L., C. Tegner, R. G. Cawthorn, F. J. Kruger, and J. R. Wilson (2006), Trapped intercumulus liquid in the main zone of the eastern bushveld complex, south africa, Contributions to Mineralogy and Petrology, 151(3), 352-369.

Martín-Hernández, F., and E. C. Ferré (2007), Separation of paramagnetic and ferrimagnetic anisotropies: A review, Journal of Geophysical Research: Solid Earth, 112(B3).

McEnroe, S., J. Skilbrei, P. Robinson, F. Heidelbach, F. Langenhorst, and L. Brown (2004), Magnetic anomalies, layered intrusions and mars, Geophysical Research Letters, 31(19).

Nikolaisen, E. S., R. Harrison, K. Fabian, and S. A. McEnroe (2020), Hysteresis of natural magnetite ensembles: Micromagnetics of silicate-hosted magnetite inclusions based on focused-ion-beam nanotomography, Geochemistry, Geophysics, Geosystems, p. e2020GC009389.

Ó Conbhuí, P., W. Williams, K. Fabian, P. Ridley, L. Nagy, and A. R. Muxworthy (2018), MERRILL: Micromagnetic Earth related robust interpreted language laboratory, Geochemistry, Geophysics, Geosystems, 19, doi:10.1002/2017gc007279.

Pastore, Z., N. S. Church, and S. A. McEnroe (2019), Multistep Parametric Inversion of Scanning Magnetic Microscopy Data for Modeling Magnetization of Multidomain Magnetite, Geochemistry Geophysics Geosystems, 20(11), 5334-5351, doi: 10.1029/2020GC009580.

Reiche, M., and H.-J. Bautsch (1985), Electron microscopical study of garnet exsolution in orthopyroxene, Physics and Chemistry of Minerals, 12(1), 29-33.

Renne, P. R., G. R. Scott, J. M. Glen, and J. M. Feinberg (2002), Oriented inclusions of magnetite in clinopyroxene: Source of stable remanent magnetization in gabbros of the messum complex, namibia, Geochemistry, Geophysics, Geosystems, 3(12), 1-11.

Richter, C., and B. A. van der Pluijm (1994), Separation of paramagnetic and ferrimagnetic susceptibilities using low temperature magnetic susceptibilities and comparison with high field methods, Physics of the Earth and Planetary Interiors, 82(2), 113-123.

Scoates, J. S., C. J. Wall, R. M. Friedman, D. Weis, E. A. Mathez, and J. A. VanTongeren (2021), Dating the bushveld complex: Timing of crystallization, duration of magmatism, and cooling of the world's largest layered intrusion and related rocks, Journal of Petrology, 62(2), egaa107. 
Sobolev, P. (1990), Orientation of acicular iron-ore mineral inclusions in plagioclase, International Geology Review, 32(6), 616-628.

Stacey, F. D., and S. K. Banerjee (1974), The physical principles of rock magnetism, Elsevier, Amsterdam.

Suuronen, J.-P., and M. Sayab (2018), 3d nanopetrography and chemical imaging of datable zircons by synchrotron multimodal x-ray tomography, Scientific reports, $8(1), 1-13$.

Sørensen, B. E., J. Hjelen, H. W. Ånes, and T. Breivik (2020), Recent features in ebsd, including new trapezoidal correction for multi-mapping, IOP Conference Series: Materials Science and Engineering, 891, 012,021, doi:10.1088/1757-899x/891/1/012021.

Tegner, C., and R. G. Cawthorn (2010), Iron in plagioclase in the bushveld and skaergaard intrusions: implications for iron contents in evolving basic magmas, Contributions to Mineralogy and Petrology, 159(5), 719-730.

Thormann, L., B. Buchspies, C. Mbohwa, and M. Kaltschmitt (2017), Pge production in southern africa, part i: Production and market trends, Minerals, 7(11), 224.

Usui, Y., T. Shibuya, Y. Sawaki, and T. Komiya (2015), Rock magnetism of tiny exsolved magnetite in plagioclase from a paleoarchean granitoid in the pilbara craton, Geochemistry, Geophysics, Geosystems, 16(1), 112-125.

Wager, L. R., and G. M. Brown (1967), Layered igneous rocks, WH Freeman.

Webb, S. J., L. D. Ashwal, and R. G. Cawthorn (2011), Continuity between eastern and western bushveld complex, south africa, confirmed by xenoliths from kimberlite, Contributions to Mineralogy and Petrology, 162(1), 101-107.

\section{A: Additional Data and Figures}

The here used list of approximately equi-distributed Fibonacci directions on the upper hemisphere $z>0$ can be calculated in Mathematica by

$\mathrm{FN}=20 ;$

XYZ[lon_, lat_] := $\{\operatorname{Cos}[1 \mathrm{lon}] \operatorname{Cos}[\mathrm{lat}], \operatorname{Sin}[\mathrm{lon}] \operatorname{Cos}[\mathrm{lat}], \operatorname{Sin}[\mathrm{lat}]\}$;

Table $[X Y Z[2 \mathrm{Pi} \mathrm{k} /$ GoldenRatio, $\operatorname{ArcSin}[2 \mathrm{k} /(2 \mathrm{FN}+1)]],\{\mathrm{k}, 1 ., \mathrm{FN}\}]$ 
Table A.1. The remanence vector directions of each silicate for the 20 applied external field directions (labeled in Fig. 9a for orthopyroxene and 9b for plagioclase) during MERRILL modelling. The following matrix show the conversion of axes between the figures (Py) and the calculations in MERRILL, where the axes in MERRILL $=$ mesh $=$ FIB .

$$
\left(\begin{array}{l}
X_{P y} \\
Y_{P y} \\
Z_{P y}
\end{array}\right)=\left(\begin{array}{ccc}
1 & 0 & 0 \\
0 & 0 & 1 \\
0 & -1 & 0
\end{array}\right)\left(\begin{array}{l}
X_{M e r r} \\
Y_{M e r r} \\
Z_{M e r r}
\end{array}\right)
$$

\begin{tabular}{l|ccc|ccc|ccc} 
Direction & \multicolumn{2}{|c|}{ External field } & \multicolumn{3}{|c}{ Orthopyroxene } & \multicolumn{3}{c}{ Plagioclase } \\
\hline \multirow{2}{*}{1} & $F I B_{X}$ & $F I B_{Y}$ & $F I B_{Z}$ & $F I B_{M X}$ & $F I B_{M Y}$ & $F I B_{M Z}$ & $F I B_{M X}$ & $F I B_{M Y}$ & $F I B_{M Z}$ \\
2 & -0.736 & -0.675 & 0.049 & -0.346 & -0.562 & 0.359 & -0.306 & -0.382 & 0.027 \\
3 & 0.087 & 0.99 & 0.097 & -0.108 & 0.026 & -0.002 & -0.082 & 0.044 & 0.022 \\
4 & 0.602 & -0.785 & 0.146 & -0.020 & -0.180 & 0.104 & 0.058 & -0.100 & -0.053 \\
5 & -0.965 & 0.171 & 0.195 & -0.105 & -0.146 & 0.100 & -0.047 & 0.014 & 0.161 \\
6 & 0.818 & 0.521 & 0.244 & -0.015 & -0.005 & 0.008 & 0.024 & 0.088 & 0.012 \\
7 & -0.248 & -0.923 & 0.293 & -0.067 & -0.098 & 0.067 & -0.022 & 0.004 & 0.016 \\
8 & -0.433 & 0.834 & 0.341 & -0.081 & 0.001 & 0.012 & -0.065 & 0.061 & 0.050 \\
9 & 0.864 & -0.316 & 0.390 & -0.049 & -0.072 & 0.049 & -0.012 & 0.008 & 0.023 \\
10 & -0.831 & -0.343 & 0.439 & -0.082 & -0.127 & 0.084 & -0.046 & -0.031 & 0.029 \\
11 & 0.369 & 0.790 & 0.488 & -0.038 & -0.057 & 0.039 & -0.028 & 0.009 & 0.047 \\
12 & 0.253 & -0.805 & 0.537 & -0.024 & -0.109 & 0.066 & -0.011 & -0.031 & 0.046 \\
13 & -0.701 & 0.406 & 0.585 & -0.048 & -0.085 & 0.056 & -0.038 & 0.003 & 0.064 \\
14 & 0.755 & 0.166 & 0.634 & -0.029 & -0.118 & 0.073 & -0.020 & 0.026 & 0.075 \\
15 & -0.420 & -0.596 & 0.683 & -0.053 & -0.151 & 0.094 & -0.039 & -0.001 & 0.076 \\
16 & -0.087 & 0.676 & 0.732 & -0.060 & -0.102 & 0.067 & -0.055 & 0.026 & 0.090 \\
17 & 0.478 & -0.403 & 0.780 & -0.046 & -0.132 & 0.083 & -0.039 & -0.0004 & 0.088 \\
19 & -0.558 & -0.023 & 0.829 & -0.064 & -0.158 & 0.099 & -0.058 & 0.018 & 0.099 \\
\hline 0 & 0.339 & 0.337 & 0.878 & -0.050 & -0.171 & 0.106 & -0.048 & 0.037 & 0.107 \\
\hline 19 & -0.017 & -0.375 & 0.927 & -0.059 & -0.193 & 0.119 & -0.053 & 0.015 & 0.108 \\
& -0.141 & 0.168 & 0.975 & -0.074 & -0.211 & 0.132 & -0.642 & 0.034 & 0.117
\end{tabular}


Table A.2. The total hysteresis properties calculated by summarizing the individual hysteresis loops for all particles in each external field directions visualized for plagioclase in Fig. 5a and for orthopyroxene in Fig. 5b.

\begin{tabular}{|c|c|c|c|c|c|c|}
\hline \multirow[t]{2}{*}{ Direction } & \multicolumn{3}{|c|}{ Orthopyroxene } & \multicolumn{3}{|c|}{ Plagioclase } \\
\hline & $M_{r s} / M_{s}$ & $H_{c}(\mathrm{mT})$ & $\chi^{*}(\mathrm{SI})$ & $M_{r s} / M_{s}$ & $H_{c}(\mathrm{mT})$ & $\chi^{*}(\mathrm{SI})$ \\
\hline 1 & 0.616 & 15.692 & 15.4 & 0.445 & 34.569 & 4.1 \\
\hline 2 & 0.561 & 22.228 & 8.4 & 0.392 & 41.164 & 4.2 \\
\hline 3 & 0.548 & 15.029 & 16.6 & 0.325 & 34.363 & 3.8 \\
\hline 4 & 0.380 & 9.562 & 15.3 & 0.372 & 28.063 & 4.9 \\
\hline 5 & 0.452 & 15.592 & 12.3 & 0.371 & 31.121 & 4.0 \\
\hline 6 & 0.709 & 17.448 & 14.9 & 0.454 & 45.037 & 4.0 \\
\hline 7 & 0.407 & 18.847 & 9.68 & 0.477 & 40.971 & 4.4 \\
\hline 8 & 0.413 & 12.412 & 15.8 & 0.198 & 23.342 & 3.6 \\
\hline 9 & 0.603 & 13.659 & 17.6 & 0.412 & 25.873 & 5.8 \\
\hline 10 & 0.338 & 28.743 & 4.5 & 0.392 & 35.929 & 4.3 \\
\hline 11 & 0.684 & 17.030 & 14.7 & 0.371 & 46.593 & 3.5 \\
\hline 12 & 0.284 & 10.143 & 10.9 & 0.510 & 29.970 & 5.1 \\
\hline 13 & 0.231 & 13.722 & 9.0 & 0.236 & 22.394 & 4.3 \\
\hline 14 & 0.725 & 17.553 & 14.7 & 0.453 & 30.647 & 4.7 \\
\hline 15 & 0.153 & 32.254 & 2.7 & 0.518 & 39.469 & 4.5 \\
\hline 16 & 0.516 & 17.993 & 12.2 & 0.272 & 30.492 & 3.3 \\
\hline 17 & 0.484 & 18.105 & 10.2 & 0.464 & 24.328 & 5.5 \\
\hline 18 & 0.132 & 27.471 & 2.7 & 0.416 & 35.198 & 4.7 \\
\hline 19 & 0.565 & 21.852 & 8.9 & 0.362 & 29.469 & 4.6 \\
\hline 20 & 0.304 & 33.737 & 3.5 & 0.488 & 31.278 & 5.2 \\
\hline
\end{tabular}




\section{External field direction locations} $-Z$

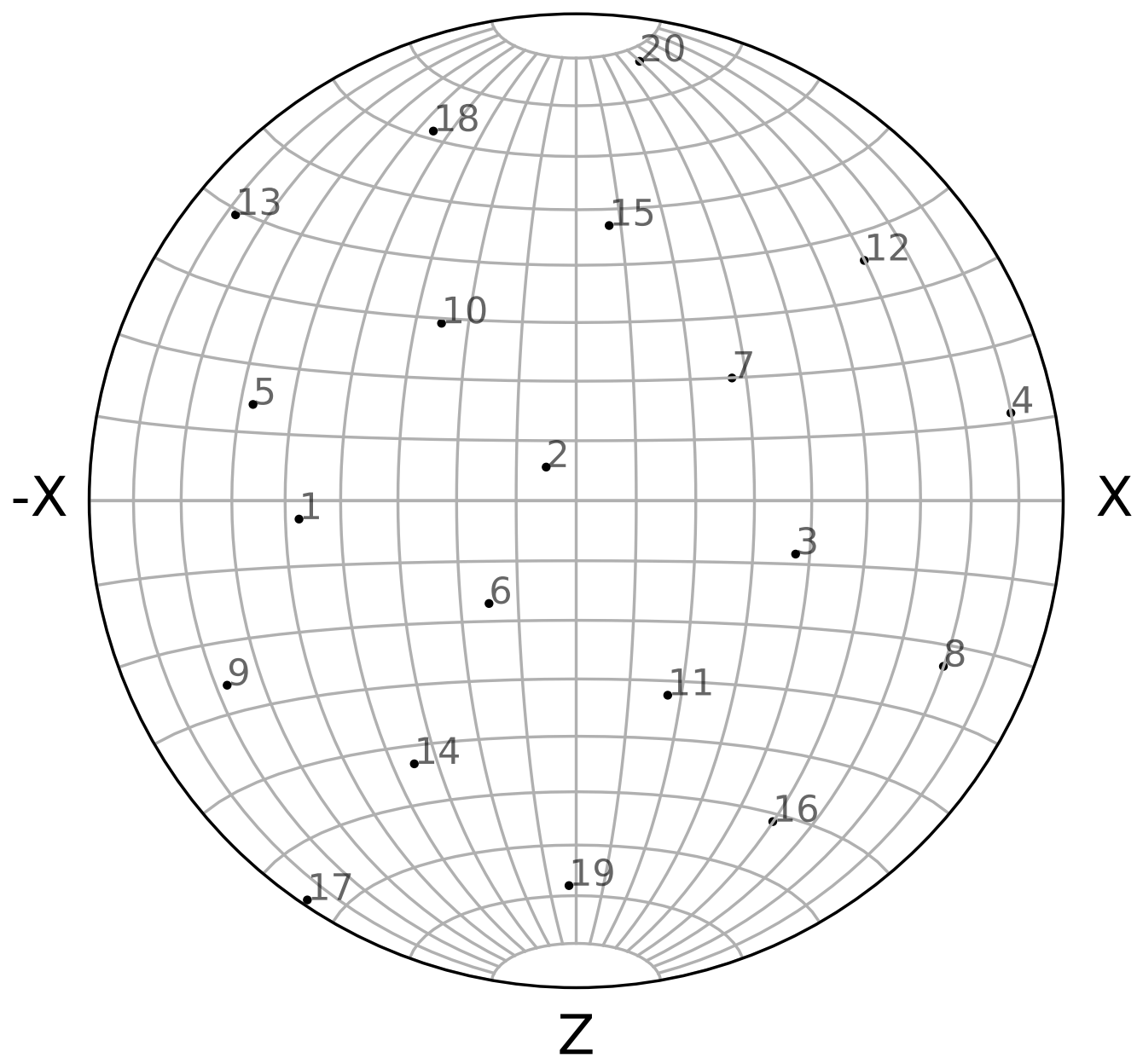

Figure A.1. Stereographic projection showing the location for each external field directions as seen in Fig. 8 . 


\section{Reflected light microscopy in oil at $125 \mathrm{X}$}
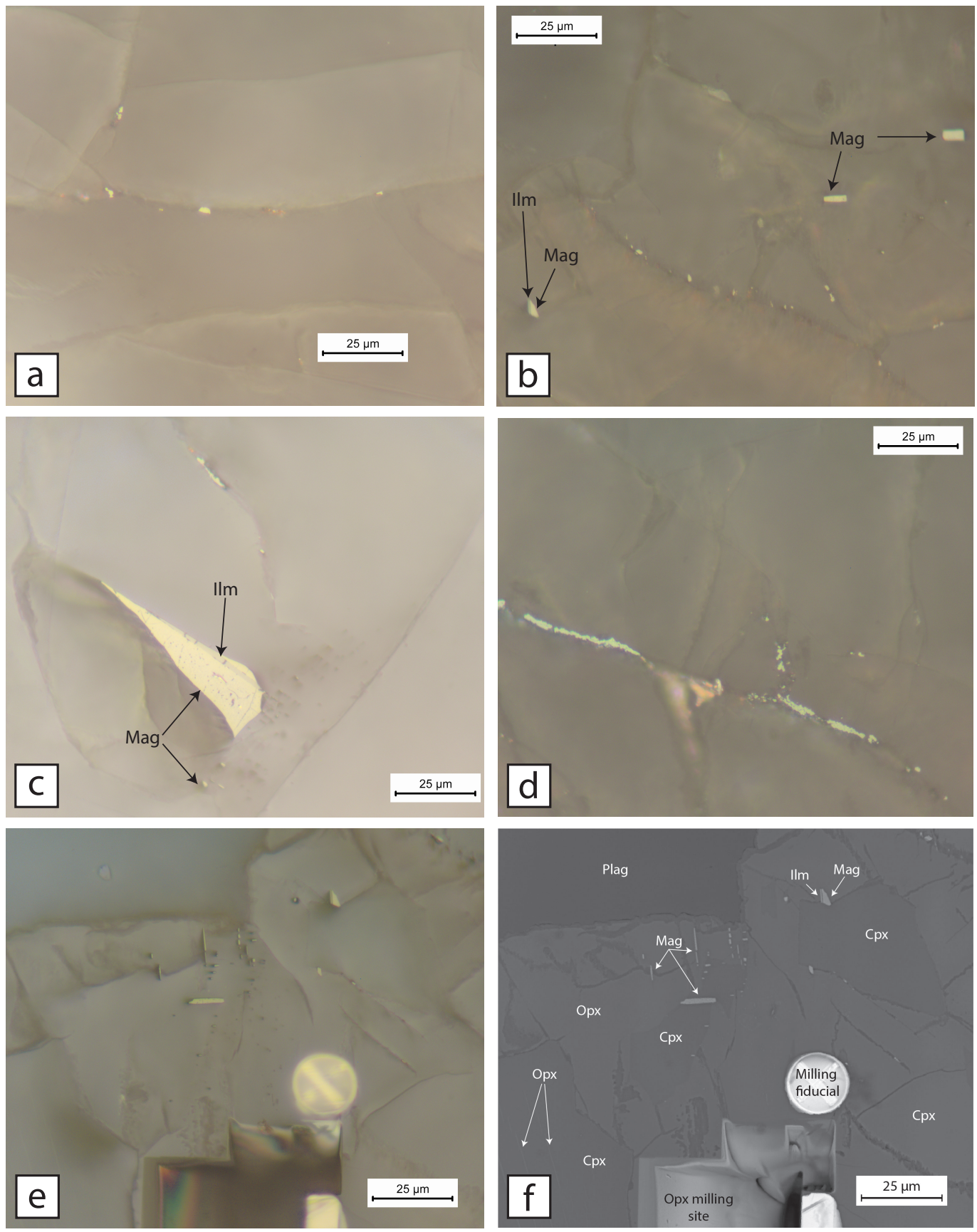

Figure A.2. Optical images taken at $125 \times$ in oil. (a) Grain boundary Fe-oxides, (b) magnetite crystallographic inclusions with ilmenite lamellae and grain boundary Fe-oxides. (c) large crystallographic oriented magnetite inclusion with ilmenite lamellae next to magnetite lamellae. (d) crack filling Fe-oxides with carbonate in the orthopyroxene. (e) patch of magnetite lamellae intersected by the milled orthopyroxene volume (Fig. 1c and 3). (f) BSD of the same location as in (e), showing the clinopyroxene exsolution and the shape differences of the magnetite when hosted by different pyroxenes. 


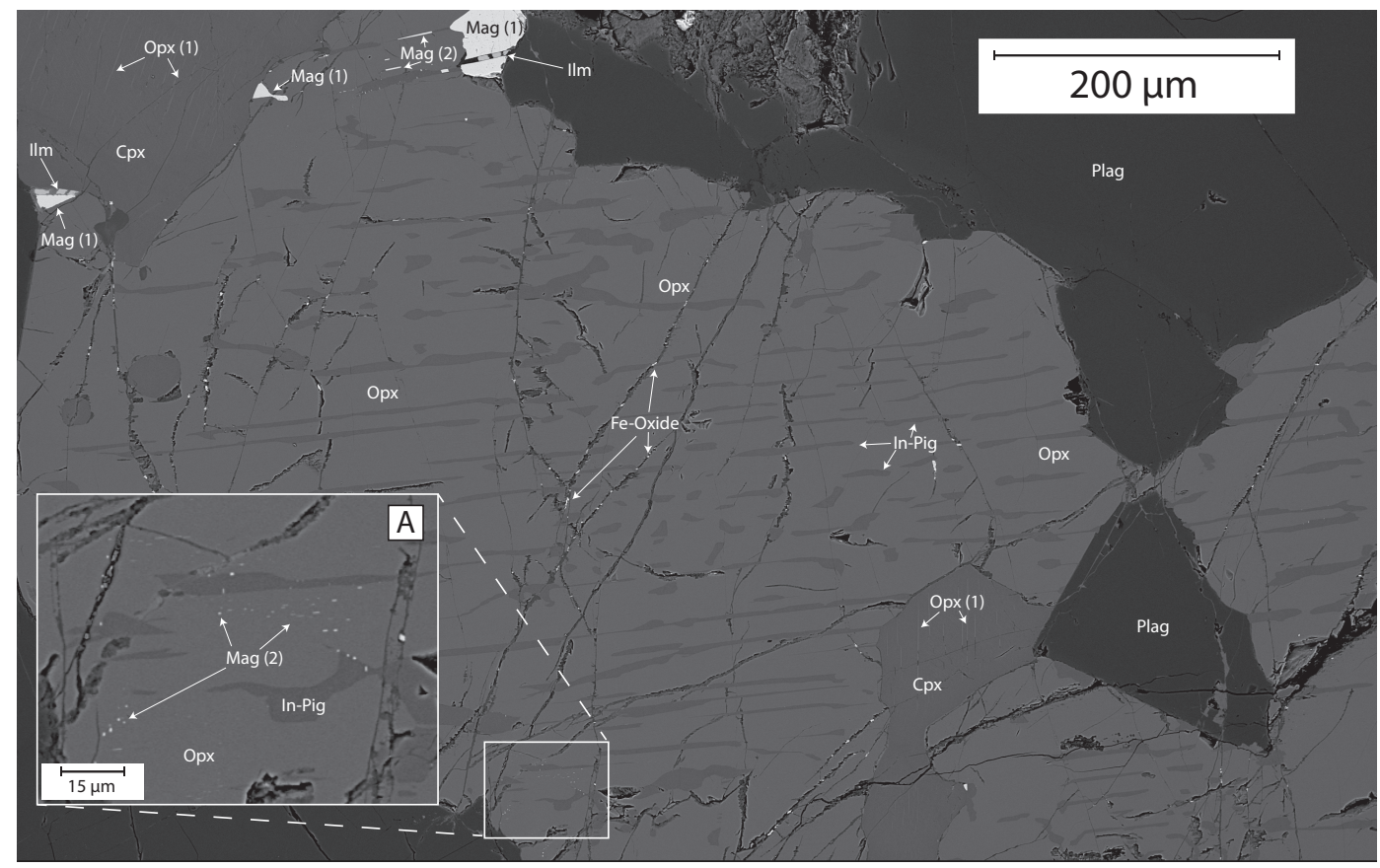

Figure A.3. BSD image of the general pyroxene crystals, dominated by orthopyroxene (Opx). The orthopyroxene contain discrete clinopyroxene crystals (Cpx) and esxolution lamellae (In-Pig) of inverted pigeonite. The discrete clinopyroxene crystals have exsolution lamellae of orthopyroxene $(\mathrm{Opx}(1))$ and Alspinel. Magnetite $(\operatorname{Mag}(1))$ are observed as crystallographic oriented inclusions $(50-100 \mu \mathrm{m})$ with ilmenite lamellae (Ilm), as exsolution lamellae (Mag 2) and grain boundary inclusions (Fig. A.2a and b). Fe-oxides occur as crack infill (Fe-Oxide and Fig. A.2d). 


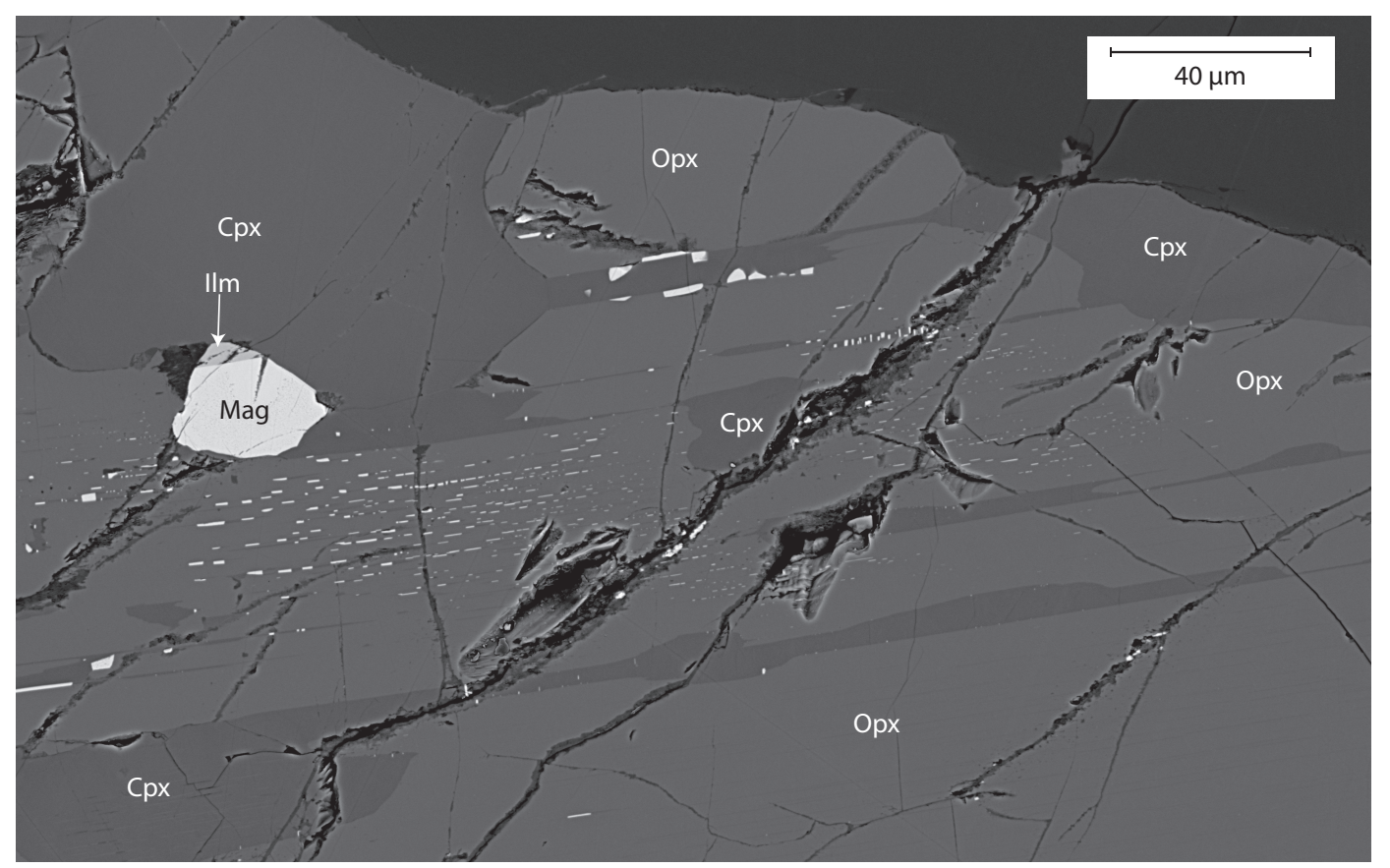

Figure A.4. Patch of concentrated magnetite lamellae oriented in the $[001]_{\mathrm{opx}}$ direction. 


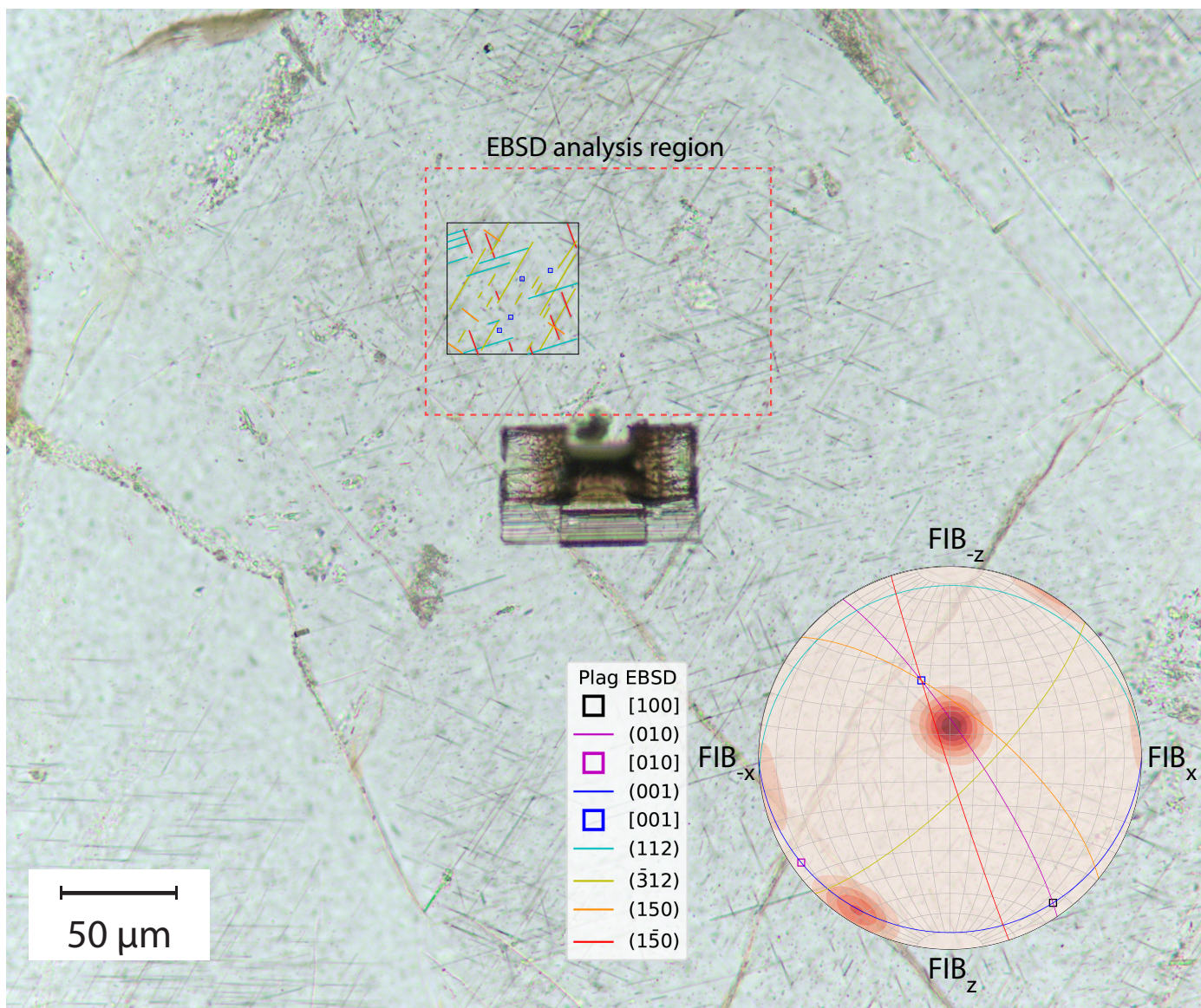

Figure A.5. Optical image of the milling site in the plagioclase with overlay of the EBSD stereonet.

Each documented direction of magnetite exsolutions from other publications that we observed are indicated with a line in the same color as the crystallographic orientation the magnetite is expected to populate. The magnetite exsolutions that are sub-parallel to the $[001]_{\text {plag }}$ is indicated at the centre of the blue squares. The red contours show the population of elongation axes from the milling volume (Fig. 2). 

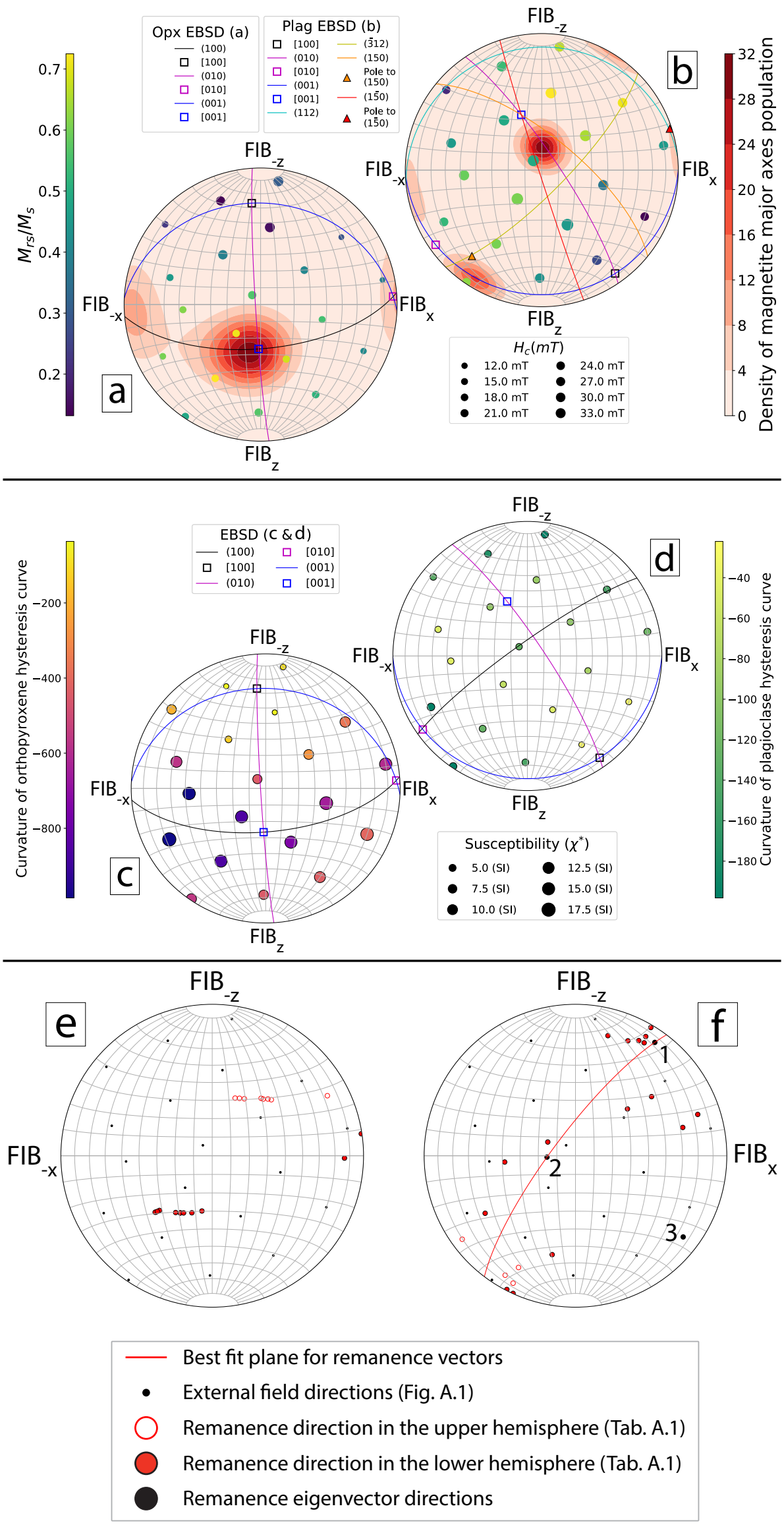

Figure A.6. A single collective figure of Fig. 8,10 and 9. 


\begin{tabular}{|c|c|c|c|}
\hline Rand & tion & $\mathrm{Max}_{\mathrm{c}}$ & $\operatorname{Max} M_{r s} / M_{s}$ \\
\hline $\begin{array}{l}\text { PLAG059 } \\
\\
\Delta y \\
z \quad x \\
\end{array}$ & $\begin{array}{r}\sum_{\text {Down }}^{\text {Up }} \\
450 \mathrm{~nm} \\
\end{array}$ & \#F: 20 & xy \\
\hline $\begin{array}{c}\text { PLAG091 } \\
\Delta x \times \\
z\end{array}$ & $150 \mathrm{~nm}$ & \#F: 14 & \#F: 16 \\
\hline $\begin{array}{l}\text { PLAG045 } \\
\text { \&x } \\
z=\end{array}$ & $200 \mathrm{~nm}$ & $\mathrm{C}^{\left(4 x^{2}\right.}$ & \#F: 14 \\
\hline
\end{tabular}

Figure A.7. Figure of micromagnetic models of the different domain states in plagioclase. The random minimization state (left column) is the lowest of 20 minimizations started from a random seed. The maximum $H_{c}$ (middle column) and ma $M_{r s} / M_{S}$ (right column) states are saturation remanence states calculated from the hysteresis loops for individual external field direction (\#F) that yielded the maximum coercivity and saturation magnetization, respectively (Fig. A.1). The $\mathrm{FIB}_{x}$ and $\mathrm{FIB}_{y}$ direction of \#F is visually represented as a stereographic projection of the upper hemisphere, for each particle by the arrow at the bottom left corner. All external fields have a positive $\mathrm{FIB}_{z}$ direction oriented out of the page. 